in: Lachièze-Rey, Marc (éd.), L'espace physique, entre mathématiques et philosophie, Collection «Penser avec les sciences », EDP-Sciences, Paris, 2006, p. 41-79.

\title{
L'espace physique vu du monde quantique : une approche épistémologique
}

\author{
Michel PATY ${ }^{\mathrm{X}}$
}

RESUME.- La pensée physique - et, jusqu'à un certain point, la pensée mathématique, du moins géométrique - s'est toujours appuyée sur la notion d'espace, comme par exemple le concept de dimension spatiale qui a donné l'idée de grandeur physique mathématisée. Jusqu'à la théorie de la relativité générale, celle-ci comprise, les conceptions des systèmes physiques et des phénomènes dont ils sont le siège ou l'origine ont été tributaires d'une pensée de l'espace, et ces phénomènes et ces systèmes sont, en général, pensés dans l'espace, même s'ils ont contribué à modifier la pensée de l'espace et s'ils ont obligé à reformuler notre «intuition spatiale». La physique quantique a remis en question cette certitude tranquille en mettant la pensée de l'espace et dans l'espace devant le dilemme suivant : ou bien garder le concept d'espace physique dans le sens précédent qui nous semble naturel et renoncer à l'idée que les «systèmes quantiques» pourraient être représentés directement dans cet espace comme des systèmes dotés de propriétés qui leur soient propres ; ou bien admettre que cela a un sens de parler de systèmes quantiques ayant des propriétés et enlever à l'espace son rôle premier dans la représentation de ces systèmes.

En prenant ce second point de vue, on est amené à penser la physique autrement que «dans l'espace» au sens habituel ; et cependant, on a toujours, à un moment ou à un autre, à envisager les systèmes physiques quantiques en référence à cet espace, qui est celui des corps macroscopiques et du cosmos dans son état actuel. Se demander comment une telle pensée est possible amène donc en même temps à s'interroger sur le rapport entre le monde quantique et l'espace physique de notre expérience. Cette interrogation passe par un inventaire des modifications du concept d'espace requises par la description physique des systèmes quantiques. On concluera en évoquant l'idée d'une émergence du concept d'espace à partir des phénomènes quantiques, tant du point de vue épistémologique (émergence d'un certain niveau de réalité à partir de niveaux sous-jacents) que $\mathrm{du}$ point de vue cosmogénétique (formation et expansion de l'espace physique des objets macroscopiques).

\footnotetext{
${ }^{x}$ Equipe REHSEIS (UMR 7596), CNRS et U niversité Paris 7-Denis Diderot, Centre Javelot, F- 75251 Paris-Cedex 05. Courriél : paty@paris7.jussieu.fr
} 


\section{ABSTRACT.- Physical space as seen from the quantum world. An epistemological approach.}

Physical thought - and, up to a certain point, mathematical thought, at least geometrical one has always leaned upon the notion of space, as for example, the concept of spacial dimension that has given rise to the idea of mathematically expressed physical magnitude. Up to the general relativity theory, and including it, the conceptions about physical systems and their generated phenomena have been dependent on a thinking of space, and these phenomena and systems are, in general, thought in space, even if they have contributed to modify the ideas about space and if they have obliged to reformulate our "spacial intuition». Quantum physics has challenged this quiet assurance by putting the thinking of space and in space in front of the following dilemma : either to keep the concept of physical space in the preceding sense, that seems natural to us, and to give up the idea that «quantum systems» could be represented directly in this space as systems endowed with properties of their own; either to admit that it is meaningful to speak of quantum systems having properties, and take away the primacy of space in the representation of these systems.

By taking this second point of view, one is led to think physics differently than «in space» in the usual sense, and nevertheless one will always have, at some time or another, to consider quantum physical systems by referring them to this space, that is the one of macroscopic bodies and of the cosmos in its present stage. To wonder how such a thought is possible brings forth therefore, at the same time, to question the relationship between the quantum world and the physical space of our experience. For such a wondering it is needed to inventory the modifications of the concept of space that are required by the physical description of quantum systems. We shall conclude by evoking the idea of an emergence of the concept of space from quantum phenomena, from the epistemological point of view (emergence of a certain level of reality from underlying levels) on the one hand, as well as from the cosmogenetic point of view (formation and expansion of the physical space of macroscopic objects) on the other hand.

\section{TABLE}

1. Introduction.

2. Penser l'espace et penser dans l'espace

3. Caractérisation d'un "monde quantique"

4. La physique quantique et le concept "classique" d'espace.

5. Le "pré-espace" physique quantique et l'espace physique classique.

1.

INTRODUCTION.

Il me faut, en premier lieu, expliciter un tant soit peu ou définir sommairement ce que j'entends par chacune des deux expressions figurant dans le titre de cet exposé : "L'espace physique vu du monde quantique", quitte à y revenir ensuite avec plus de détail.

Prenons, d'abord, la première expression, qui est la plus familière, l'espace physique. J'entends, par espace physique, l'espace de notre expérience du comportement des objets macroscopiques (y compris notre propre corps), qui est aussi l'espace de la physique macroscopique, et qui peut être décrit soit comme 
espace des sensations et de la perception, soit comme espace géométrique (topologique et métrique), reconstruit intellectuellement à partir du précédent, et indépendant du sujet de la connaissance. C'est sur cet espace de la géométrie que la physique classique puis relativiste a fondé ses élaborations, le prenant pour cadre ou siège des phénomènes physiques, et on le considère généralement, en conséquence, comme étant l'espace physique : nous reviendrons sur ses divers caractères, acquis au cours de ces élaborations.

Considérons maintenant la seconde expression. Parler d'un monde quantique (ou d'un domaine quantique: celui des phénomènes ou des objets quantiques), cela veut dire accorder aux éléments d'un tel monde qu'ils possèdent une consistance propre, ce qui peut aussi se dire autrement : qu'ils possèdent des propriétés physiques, ou encore qu'ils sont réels. C'est cela qui est nié généralement, notamment par la position qui correspond à l'interprétation philosophique de la mécanique quantique connue comme celle de l'Ecole de Copenhague: selon cette vue, les propriétés des systèmes quantiques sont inéluctablement tributaires des moyens de leur approche par les appareils classiques de mesure, eux-mêmes fondés sur les concepts et les théories de la physique classique ${ }^{1}$.

Admettre que l'on puisse légitimement parler d'un monde (de propriétés) quantiques, cela suppose qu'il soit possible de se placer d'un point de vue proprement quantique, par abstraction du sujet perceptif et des moyens et dispositifs qui lui fournissent les éléments de perception sensible à partir desquels il établit une connaissance intellectuelle de ce domaine ou de ce monde. Cela suppose que l'on puisse considérer une description théorique de ce domaine qui soit à la fois physique et auto-consistante, c'est-à-dire que, d'une part, elle se rapporte (exprimée, bien entendu, au moyen de représentations symboliques) aux phénomènes concernés du monde physique (le monde réel, tel qu'on l'appelle communément); et que, d'autre part, l'on n'ait pas besoin de recourir pour elle à un référent externe tel que celui de l'observation ou de la perception, qui sont anthropocentriques. Il faut donc supposer que l'on dispose d'une théorie propre ou intrinsèque du domaine quantique, et sortir, évidemment, du «cercle» de l'interprétation observationaliste ou «complémentariste», en adoptant par principe une perspective réaliste sur le monde physique et sur la théorie physique comme connaissance effective de ce domaine de la nature. Nous reviendrons également sur cette question préliminaire et fondamentale.

Le problème que je voudrais tenter d'éclairer du point de vue épistémologique est celui du rapport entre ces deux notions, en les supposant fondées : espace physique, monde quantique. En particulier, nous savons que les phénomènes et les systèmes quantiques impliquent une critique de l'espace tel que la physique classique et relativiste le considère, par exemple, l'espace lié au temps

${ }^{1}$ Bohr [1958]. 
de la théorie de la relativité (restreinte ou générale). Dans cette théorie, les systèmes physiques sont toujours considérés comme sous-tendus par la notion de points matériels individuels localisés dans cet espace-temps, liés entre eux par une relation de causalité spatio-temporelle: en relativité restreinte, deux points physiquement, c'est-à-dire causalement, reliés ou susceptibles de l'être, appartiennent à une même région de «type temps» du cône de lumière, telle que $c^{2} t^{2}-r^{2} \geq 0$ ). Les systèmes «classiques" (dans ce sens, c'est-à-dire non quantiques) étendus sont tels que leurs structure ponctuelle élémentaire est toute entière située dans cette région de type temps (par exemple le front d'une onde sonore, le front d'une onde lumineuse ${ }^{2}$, ou un bâton rigide).

La critique de l'espace physique par la physique quantique est portée par les relations d'indétermination (distribution spectrale des variables conjuguées d'espace et d'impulsion, $\Delta x . \Delta p>\hbar$ ), par la dualité onde-corpuscule (diffraction, interférences, qui impliquent une extension dans l'espace des « objets physiques » considérés), par la non-localité des systèmes quantiques, par la non-séparabilité locale de systèmes ayant interagi dans le passé, par l'indiscernabilité des «particules» identiques (fermions ou bosons) composant un système quantique. Qui dit ici critique de l'espace, dit en réalité critique du concept d'objet-dansl'espace $^{3}$. Nous nous interrogerons, en particulier, sur la réponse traditionnelle de l'interprétation orthodoxe de la mécanique quantique en termes d'approche statistique, et sur la signification à cet égard de la connaissance, relativement récente, de systèmes quantiques individuels.

Que devient donc le concept d'espace de la géométrie et de la physique des corps matériels et des champs classiques avec la physique quantique? En demeure-t-il quelque chose après cette critique qui revient à limiter ses conditions de validité, et peut-être même sa signification ? On doit se rappeler que notre concept d'espace physique nous vient de notre connaissance des propriétés des objets macroscopiques (non quantiques) et que rien ne nous assure qu'il soit encore pertinent dans le domaine atomique et infra-atomique ${ }^{4}$; mais que, d'un autre coté, l'élaboration et la formulation de la physique quantique continue de faire appel à ce concept, non seulement comme concept générateur (voir la façon dont les opérateurs quantiques pour représenter les variables dynamiques sont construits dans la théorie, en particulier celui de position) ${ }^{5}$, mais aussi dans son

\footnotetext{
${ }^{2}$ Les points d'un front d'onde lumineuse sont reliés causalement à leur commune origine, et donc sont situés sur le cône de lumière de l'espace-temps.

${ }^{3}$ Einstein lui-même reconnaissait que la physique quantique "ne se propose aucunement de donner une représentation mathématique en termes d'espace et de temps" (Einstein [1940]). Voir Paty [1988], chap. 5, p. 188-193 ; [à paraître, a], chap. 6 .

${ }^{4}$ Un collaborateur de l'Encyclopédie de Diderot et d'Alembert, M. Guénault, le rappelait au XVIII è siècle (Guénault [1756]; cf. Paty [1998b]). Une telle idée a été également émise à propos des phénomènes quantiques, entre autres par Paul Langevin (Langevin [1934]) ; Einstein lui-même en a évoqué la possibilité (voir notamment : Einstein [1933, 1936, 1940]).

${ }^{5}$ Dirac [1930]. Cf. Paty [1985].
} 
sens le plus classique.

A cet égard, on doit accorder une attention privilégiée à la réflexion sur la notion de propagation d'un système quantique dans l'espace, en rapport au principe de superposition, à la question de la dispersion ou de l'étalement (comme pour une onde), ainsi qu'à celle de la cohérence d'un système quantique isolé dans l'espace. On se demandera aussi comment se caractérise ou s'engendre l'espace occupé par un système purement quantique de relativement grandes dimensions tel qu'un condensat de Bose-Einstein. Nous ne prétendons pas apporter une solution à de telles questions, mais seulement les approcher sous l'éclairage épistémologique, qui est celui de leur signification possible.

\section{2.}

\section{PENSER L'ESPACE ET PENSER DANS L'ESPACE}

\section{La physique tributaire des notions d'espace et de géométrie}

La pensée physique - et, jusqu'à un certain point, la pensée mathématique, du moins géométrique - s'est toujours appuyée sur la notion d'espace. Celle-ci a toujours été en même temps au centre de la réflexion philosophique, de la théorie aristotélicienne des lieux naturels à l'espace homogène et isotrope de la géométrie et de la physique de Galilée, Descartes, Huygens, Newton, Leibniz, et jusqu'à la physique contemporaine du continuum spatio-temporel. Songeons, pour nous en tenir à la période ouverte par la révolution scientifique du XVII è siècle, à la conception des idées innées de Descartes qui accompagna sa Géométrie, à sa critique par Locke pour qui l'origine des idées se trouve dans les sensations, à l'esthétique transcendantale de Kant (l'espace et le temps comme formes intuitives a priori de la sensibilité), aux analyses épistémologiques faites au XIX è siècle par Hermann von Helmholtz, Ernst Mach, William James, Henri Poincaré, fondées en partie sur les études psycho-physiologiques, et enfin à celles, au XX è, que l'on doit à Albert Einstein, Hermann Weyl, Ernst Cassirer, Rudolf Carnap, Hans Reichenbach et d'autres, sans oublier les travaux des psychologues et des neurophysiologues, de Jean Piaget à Jean-Pierre Changeux, Alain Berthoz....

Nous en retiendrons ici que la notion d'espace s'est constituée dans un premier temps comme "espace perceptif" et "représentatif" à partir des données de la perception, qui tiennent à notre constitution physiologique et neuropsychologique mettant en jeu les différents sens (du toucher, de la vison, de l'ouie, ...) ; cet "espace représentatif", obtenu par la coordination des différents espaces perceptifs, n'est ni homogène et isotrope ni même à trois dimensions, si 
ces dimensions sont à rapporter aux différents paramètres qui font la perception ${ }^{6}$. On peut cependant considérer, avec Mach, que ces dimensions sont d'origine à la fois physique et physiologique, résultant de l'organisation spatiale des organismes vivants supérieurs eux-mêmes suivant les orientations naturelles dues à la pesanteur, à la locomotion (haut-bas, avant-arrière), et à une asymétrie droitegauche $^{7}$. Pour Poincaré, ces trois dimensions sont plutôt caractérisées au niveau de la coordination des espaces sensoriels, à travers une analyse de la constitution de la notion de continu, qui fonde l'“Analysis situ”, étude de l'espace (géométrique) en tant qu'il est continu et à trois dimensions ${ }^{8}$.

Quant à l'espace géométrique, qui s'est constitué par les opérations de l'entendement à partir de l'espace représentatif, il peut être considéré soit comme

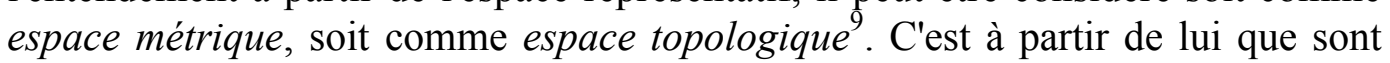
définies les grandeurs et les figures de la géométrie (avec, en premier lieu pour les grandeurs, les distances spatiales), et c'est à lui qu'est identifié, de Galilée, Descartes, Newton, à Riemann, Maxwell, Helmholtz, Poincaré (pour ne citer qu'eux), l'«espace physique». Après la reconstruction axiomatique de la Géométrie de Hilbert ${ }^{10}$, une dissociation s'est opérée entre la géométrie purement mathématique, et la géométrie de l'espace physique : Einstein est un bon témoin de cette nouvelle perspective, en définissant, dans ses deux théories de la relativité, l'espace physique par des grandeurs géométriques (coordonnées et distances) prises sur des corps physiques, moyennant des relations de correspondance ou de coordination, qui reviennent à la construction d'une "géométrie physique" élémentaire par celle d'un "espace physique de référence". Cette notion lui a été indispensable pour penser la cinématique de la relativité restreinte indépendamment de la dynamique, et pour s'affranchir, avec la relativité générale, des limitations de l'espace euclidien. ${ }^{11}$

Il faudrait compléter ces remarques en évoquant les enseignements de l'histoire des sciences qui montrent comment le concept d'espace de la physique et de la géométrie s'est progressivement élaboré, en rupture avec les conceptions communes et par construction intellectuelle, mettant en œuvre les ressources de la pensée rationnelle, mathématique et physique : des lieux aristotéliciens qualitatifs à l'espace continu homogène, isotrope et infini de Bruno, Galilée, Descartes, Gassendi, et à la pensée infinitésimale et différentielle du continu spatial, de Newton, Leibniz, Euler, d'Alembert, Lagrange, à Riemann, Ricci, Levi-Civitta.

\footnotetext{
6 Tels que les divers mouvements musculaires dirigeant le toucher ou l'orientation du globe oculaire pour la vision : voir Poincaré [1902], chapitres 3, 4 et 5.

7 Mach [1905, 1906].

${ }^{8}$ Ce terme, "Analysis situ”, inventé par Leibniz, fut repris par Riemann pour désigner la topologie, qu'il fonda. Voir Poincaré [1905], chapitre 4 ; [1913], chapitre 3.

${ }^{9}$ Voir Paty [1993], chapitres 6 et 7 ; [1998b].

${ }^{10}$ Hilbert [1899].

${ }^{11}$ Voir les travaux désormais classiques d'Einstein sur la relativité (Einstein [1987-], vols. 2 à 8). Voir aussi Weyl [1918], Schrödinger [1950]; cf. Paty [1993], chapitres 3, 4, 5
} 
L'espace, en physique comme en géométrie, est, depuis lors, un concept construit mathématiquement à l'aide de grandeurs appropriées à sa structure et à ses propriétés, qui concernent les entités physiques définies dans l'espace ${ }^{12}$.

L'idée de grandeur mathématique prend son point de départ dans la définition de la ligne droite continue, avec ses points, mais aussi, après Newton et Leibniz, avec ses éléments différentiels. Dans les Règles pour la direction de l'esprit (env. 1628), en particulier à la règle 14, Descartes analyse la notion de grandeur, soumise à l'ordre et à la mesure (au sens de rapports, de proportions), c'est-à-dire géométrisable ou arithmétisable, en se fondant sur la dimension spatiale prise pour archétype ${ }^{13}$. Kant, dans la Critique de la raison pure (2è éd., 1787), fait de cette même dimension spatiale le point de départ de sa description des grandeurs extensives qui, avec les intensives, appartiennent à ce qu'il appelle, dans son "Analytique transcendantale", les "Axiomes de l'intuition" et les "Anticipations de la perception", qui figurent parmi les principes synthétiques de l'entendement pur, rendant possible la connaissance et, en particulier, l'application des mathématiques aux phénomènes ${ }^{14}$.

Dans sa dissertation inaugurale de 1854, "Sur les hypothèses qui servent de fondements à la géometrie", aux trois dimensions de l'espace ou, plutôt, à l'espace à trois dimensions, dans son étude systématique des grandeurs ou variétés à $n$ dimensions, en l'entendant comme l'espace physique, dont les propriétés topologiques et métriques ne sont pas données par les propriétés générales des grandeurs, mais par les "concepts empiriques sur lesquels sont fondées les déterminations métriques de l'étendue", à savoir "le concept de corps solide et celui de rayon lumineux" ". Il précisait que ceux-ci "cessent de subsister dans l'infiniment petit", et ajoutait: "Il est donc très légitime de supposer que les rapports métriques de l'espace dans l'infiniment petit ne sont pas conformes aux hypothèses de la géométrie, et c'est ce qu'il faudrait effectivement admettre, du moment où l'on obtiendrait par là une explication plus simple des phénomènes. La question de la validité des hypothèses de la géométrie dans l'infiniment petit est liée à la question du principe intime des rapports métriques dans l'espace" $"$.

Cette remarque concerne directement notre propos: elle peut se traduire en une question sur la structure de l'espace dans les dimensions qui correspondent au domaine quantique. On sait que, concernant le domaine opposé, celui des très grandes dimensions, qui correspond en fait à l'astronomie et à la cosmologie (cette dernière n'existait pas encore comme science à l'époque),

\footnotetext{
${ }^{12}$ Cf. Paty [1998b].

${ }^{13}$ Descartes [1628]. Voir Paty [2001b].

${ }^{14}$ Kant [1781]1787.

${ }^{15}$ Riemann [1854]. Cf Paty [1993], chapitre 6.

${ }^{16}$ Riemann [1854].

${ }^{17}$ Riemann [1854].
} 
Riemann avait préparé les conditions de la pensée de l'espace par la théorie de la relativité générale. En caractérisant la différence entre la topologie et la métrique, Riemann était amené à distinguer 1'“illimité" (ce qui n'a pas de bornes), qui appartient aux rapports d'étendue (topologiques), et l'“infini", qui appartient aux rapports métriques, ce qui faisait voir d'emblée la possibilité de géométries non euclidiennes (en particulier, par la possibilité de concevoir des espaces finis sans limites (comme un plan sphérique).

De réceptacle indifférent de la physique newtonienne, le concept d'espace est ensuite revenu à l'indissociabilité d'avec les corps qu'il renferme (ce qui renouait, en un certain sens, avec une idée cartésienne), et les phénomènes dont il est le siège : l'espace (et, plus exactement, l'espace-temps) est structuré par la matière-énergie qu'il contient (leçon de la relativité générale chaque jour confirmée, notamment par la connaissance la plus précise des objets astronomiques).

Ces évocations nous rappellent que la pensée physique, et la mathématisation de la physique à travers la notion de grandeur d'expression mathématique, ont toujours été, jusqu'à la théorie de la relativité générale inclue, tributaires d'une pensée de l'espace. Elles nous rappellent que les phénomènes physiques et les systèmes (ou corps) physiques qui en sont le siège ou la source sont, en général, pensés dans l'espace, même s'ils ont contribué à modifier la pensée de l'espace et à réformer notre "intuition spatiale".

\section{Peut-on penser sans l'espace?}

Ainsi la physique pensait-elle dans l'espace et pensait-elle l'espace, comme elle pense dans le temps (première définition, selon Newton, après l'invention du temps physique par Galilée, de la mécanique, qui fut la première des sciences classiques à être mathématisée ${ }^{18}$ ), jusqu'à l'avènement de la physique quantique. La physique quantique a remis en question cette certitude tranquille en mettant la pensée de l'espace et dans l'espace devant le dilemme suivant: ou bien garder le concept d'espace physique dans le sens précédent qui peut sembler naturel (mais dont on a vu qu'il ne l'est pas plus que les autres, puisqu'il est construit) et renoncer à l'idée que les «systèmes quantiques» pourraient être représentés directement dans cet espace comme des systèmes dotés de propriétés qui leur soient propres; ou bien admettre que cela a un sens de parler de systèmes quantiques ayant des propriétés et enlever à l'espace son rôle premier dans la représentation de ces systèmes.

En prenant ce second point de vue, on est amené à penser la physique

${ }^{18}$ Voir Paty [1994a et b]. 
autrement que «dans l'espace» au sens habituel ; et cependant, on a toujours, à un moment ou à un autre, à envisager les systèmes physiques quantiques en référence à cet espace, qui est celui des corps macroscopiques et du cosmos dans son état actuel, notamment lorsqu'il faut raccorder les systèmes quantiques, considérés intrinsèquement, avec les systèmes d'observation par lesquels nous avons connaissance, même si c'est très indirectement, de leurs propriétés quantiques elles-mêmes. Se demander comment une telle pensée est possible amène donc, en même temps, à s'interroger sur le rapport entre le monde quantique et l'espace physique de notre expérience ${ }^{19}$. Cette interrogation passe par un inventaire des modifications du concept d'espace requises par la description physique des systèmes quantiques.

On peut aussi se demander, comme les corps macroscopiques sont constitués de systèmes quantiques (particules élémentaires, atomes, etc.), et comme leurs propriétés macroscopiques résultent de l'effet sous-jacent des propriétés quantiques ${ }^{20}$, et sont comme «émergentes» à leur niveau propre à partir de ces dernières, si le concept d'espace physique lui-même ne serait pas «émergent» à ce niveau ${ }^{21}$, que l'on supposerait constitué à partir de l'effet de propriétés quantiques qu'on pourrait appeler «pré-spatiales». On pourra envisager cette «émergence» de l'espace physique, à partir des propriétés des systèmes quantiques, de deux points de vue : un point de vue épistémologique, et un point de vue plus directement physique, en fait cosmogénétique.

Du point de vue épistémologique, on constate la caractérisation d'un certain niveau de constitution ou d'organisation par des concepts propres à partir d'un niveau sous-jacent, lui-même caractérisé par des concepts signifiants dans son domaine; les deux séries de concepts théoriques pour chacun des niveaux sont supposées se raccorder en principe l'une à l'autre par une correspondance, même si celle-ci n'est pas toujours acquise, soit par un défaut de connaissance, soit par une trop grande complexité des effets. Du point de vue cosmogénétique, on se préoccupe de caractériser et de formuler théoriquement la formation et l'expansion de l'espace physique des objets macroscopiques et du cosmos.

La difficulté ou l'impossibilité de penser sans l'espace est bien illustrée par le passage suivant de la Critique de la raison pure de Kant, dans 1'“Esthétique transcendantale": "L'espace est une représentation (Vorstellung) nécessaire a

\footnotetext{
${ }^{19}$ Sur le rapport, d'une manière générale, entre les pensées respectives du domaine quantique et du domaine classique, voir Paty [2000d].

${ }^{20}$ C'est un postulat que nous admettons, comme il est d'ailleurs généralement admis par les physiciens. Nous ne pouvons discuter ici les problèmes épistémologiques et philosophiques qu'il soulève : disons simplement qu'il s'appuie sur une conception moniste du monde naturel, qui ne se confond pas avec un réductionnisme de principe, que l'introduction des notions de niveaux de structuration des corps physiques et d'émergence permet d'éviter.

${ }^{21}$ Sur cette notion d'«émergence», voir Paty [1988], chap. 8, p. 283-284, et une discussion par Zahar [1994].
} 
priori qui sert de fondement à toutes les intuitions (Anschauungen) externes. On ne peut jamais se représenter qu'il n'y ait point d'espace, quoiqu'on puisse bien penser (denken) qu'il ne s'y trouve aucun objet. L'espace est donc considéré comme la condition de possibilité des phénomènes (Erscheinungen: ce qui apparait dans la perception), et non pas comme une détermination qui en dépende, et il est une représentation a priori servant nécessairement de fondement aux phénomènes externes" ${ }^{, 2}$. Remarquons en passant que l'indication selon laquelle on peut penser qu'il ne se trouve aucun objet dans l'espace indique combien la pensée kantienne de l'intuition de l'espace est tributaire de l'espace absolu newtonien (contenant de tous les corps, qui n'est pas affecté par eux). Kant en fait une forme a priori de la sensibilité, et non plus un concept mathématique ou physique. On sait que le contenu du concept newtonien d'espace (absolu) diffère de celui du concept cartésien correspondant : l'espace cartésien (relatif aux corps), constitue une propriété essentielle des corps. C'est le détachement par Newton de l'espace par rapport aux corps, et sa prééminence sur eux, traduite en antériorité, qui permet à Kant d'en faire la pierre angulaire de son édifice philosophique sur la connaissance.

Mais ce qu'il m'importe ici de souligner, c'est l'absolue nécessité, pour Kant, de l'espace pour concevoir les phénomènes. Si l'on veut, comme nous venons de le suggérer, remplir un programme pour la connaissance qui ne mette pas l'espace au premier plan, il nous faudra passer outre à l'« interdit kantien », ou alors concevoir autrement, plus indirectement, l'effet sur la connaissance de quelque intuition spatiale.

La définition kantienne du phénomène, Erscheinungen («ce qui apparait dans la perception »), limite ce dernier au domaine de la perception seule. Si l'on veut étendre la notion de phénomène en dépassant la perception directe, pour atteindre ce qui serait conçu et donné plus directement par l'entendement, et rapporté seulement de manière très indirecte (et complexe) dans la perception, on devra nécessairement sortir du schéma kantien. Remarquons, cependant, que l'on peut continuer de trouver, dans la philosophie kantienne, la trame élémentaire, relative pour ainsi dire, la «condition élémentaire de possibilité » d'une pensée rationnelle de la science. En transportant le contenu du concept newtonien d'espace dans le contenu de l'intuition sensible correspondante, Kant liait étroitement sa conception (son «esthétique transcendantale ») à la conception newtonienne, et devait encourir pour cela la critique empiriste, par exemple celle de von Helmholtz, plus tard celles de Reichenbach et de Carnap. Mais, d'un autre coté, ce qu'il constituait n'étant pas une physique (mais une "esthétique transcendantale »), sa conception échappait en un sens à cette même critique empiriste. C'est ce qui permit à Cassirer de proposer, dans ses ouvrages Substance

\footnotetext{
${ }^{22}$ Kant [1781]1786 (Esthét. transc., A24, B39), trad fr. : Kant, Oeuvres, vol. I, p. 785. Voir le commentaire que donne de ce passage Gilles-Gaston Granger dans son livre sur La pensée de l'espace (Granger [1999], p. 10).
} 
et fonction et La théorie de la relativité d'Einstein, de modifier la signification de l'a priori kantien, en "fonction de spatialité », compatible avec les constructions des mathématiques et de la physique modernes. L'inspiration fondamentale de l'esthétique transcendantale peut alors être considérée comme compatible avec une pensée critique, même radicale, de la notion d'espace.

Faisons à cet endroit une autre remarque. Que l'espace (et le temps) soi(en)t la condition de possibilité des phénomènes, même sous une forme atténuée, c'est ce qui est en cause avec la physique contemporaine. L'espace possède-t-il à cet égard un privilège sur d'autres notions liées fondamentalement à la perception ? (par exemple, des notions liées à des grandeurs intensives et non plus extensives, comme le son, la lumière, les couleurs, la chaleur, etc.). De Galilée et Descartes à Leibniz, Newton et Kant, l'espace et le temps ont un privilège sur les autres grandeurs, mais surtout en ceci qu'elles sont à l'origine de la pensée de ces dernières (cela est explicite dans la Règle 14 de Descartes mentionnée plus haut). Si l'on considère les données actuelles de la physiologie cérébrale et neuronale et de la psychologie cognitive, on peut admettre de maintenir ce privilège, au moins comme un choix de représentation fondamentale de référence. L'histoire des connaissances et des sciences fait également apparaître la représentation spatiale comme étant effectivement fondamentale et première, depuis les objets et les images de l'homme préhistorique jusqu'à la géométrie. Quant à la représentation temporelle, elle a pu sembler également une donnée première, encore que l'on puisse se demander si c'est la notion de temps ou celle de mouvement qui s'est imposée d'abord; mais cela revient en fin de compte au même (le temps peut être considéré comme une combinaison de mouvement et d'espace, comme le mouvement est une combinaison d'espace et de temps).

Concluons (provisoirement) sur ces questions en indiquant que Kant, malgré les restrictions qu'on a vues, laisse peut-être, par le rationalisme affirmé de sa philosophie, la porte ouverte sur une possibilité de dépasser, dans la connaissance, les limitations du sensible. Gilles Granger a donné à ce sujet une précision éclairante, à propos du rapport entre l'intuition sensible (forme a priori de la sensibilité), qui conditionne la possibilité de connaissance des objets, et la connaissance elle-même de ces objets, en faisant remarquer que Kant l'attribue au «caractère constructif de la mathématique en général, qui s'oppose alors à la philosophie, et de la géométrie en particulier $»^{23}$. Cette géométrie est pour Kant, «la science qui détermine synthétiquement et pourtant a priori les propriétés de l'espace $»^{24}$. D'autres, allant au-delà de Kant, parleront d'éléments rationnels de connaissance, inventés par la pensée créatrice, choisis «librement» (sans contrainte de nature logique telle qu'une «induction») par la raison pour éclairer notre expérience... ${ }^{25}$

\footnotetext{
${ }^{23}$ Granger [1999], p. 10-11.

${ }^{24}$ Kant [1781]1786.

${ }^{25}$ Entre autres, Poincaré et Einstein. Voir Paty [1999b].
} 


\section{Une physique sans l'espace est-elle possible?}

Les discussions sur la critique des conceptions kantiennes suscitées par les géométries non euclidiennes et la relativité générale avaient amené à moduler le rôle de la pensée de l'espace dans la connaissance, et à remettre en avant le primat de la notion de corps, à penser l'espace à partir d'elle. Pourtant les physiciens (ni les autres, d'ailleurs...) n'envisagent pas volontiers, en général, la perspective d'abandonner les concepts spatio-temporels dans les éléments de base des descriptions de la physique, pour peu du moins qu'ils se préoccupent du raccord de leurs théories aux phénomènes physiques directement accessibles.

On pourrait rassembler tout un florilège de déclarations d'auteurs, physiciens ou philosophes, faisant état de l'impossibilité de penser une physique sans espace (voir, par exemple, Louis de $\operatorname{Broglie}^{26}$ ), ou du moins de la difficulté à le faire.

Einstein, qui pouvait le concevoir, selon ce qu'il déclara à plusieurs reprises à propos des systèmes de la physique quantique, avouait cependant ne pas savoir comment penser ainsi ${ }^{27}$. Evoquant les problèmes de la théorie quantique des champs, il écrivait, dans "Physique et réalité »: «On a certes fait observer que l'introduction d'un continuum d'espace-temps pouvait, eu égard à la structure moléculaire de tout événement dans l'infiniment petit, être déjà considéré comme allant contre la nature. Le succès de la méthode de Heisenberg ne nous renverraitil pas à un mode de description de la nature purement algébrique ? Ne nous invitet-il pas à éliminer de la physique les fonctions continues ? Mais, dans ce cas, il faudrait également renoncer, sur le plan des principes, à faire usage du continuum d'espace-temps. Il n'est pas impensable que l'esprit humain, à force de perspicacité, finisse par découvrir des méthodes permettant de franchir ce pas. Mais, pour le moment, une telle ambition équivaut à vouloir respirer dans un local sans air. $»^{28}$

Et encore, dans une lettre à un correspondant, à propos de la possibilité d'envisager une théorie du discontinu, il exprimait la difficulté à penser ainsi dans les termes suivants: "Dans une telle théorie [du discontinu, sans rapports différentiels], il n'y a pas place pour l'espace et le temps, mais uniquement pour des nombres, des constructions numériques et des règles pour les former sur la base de règles algébriques excluant le processus limite (...). Dans une théorie algébrique de la physique (...), il sera particulièrement difficile, par exemple, de déduire un semblant d'ordre spatio-temporel (...). Je ne vois pas à quoi pourrait ressembler la structure axiomatique d'une physique comme celle-là, et je n'aime pas qu'on en parle par allusions obscures. Mais je crois tout à fait

\footnotetext{
${ }^{26}$ Par ex., De Broglie [1966], p. 62-63, cité dans Paty [1988].

${ }^{27}$ Einstein [1933, 1940], cité dans Paty [1988].

${ }^{28}$ Einstein [1936], tr. fr. in Einstein [1989-1993], vol. 5, p. 148.
} 
possible que l'évolution nous y conduise. (...) $»^{29}$.

Du coté des physiciens qui considèrent que l'on doit en venir à un dépassement des grandeurs spatiales, on notera la conception du «second» David Bohm, après son abandon du programme des variables cachées, envisageant de substituer à l'ordre selon l'espace et le temps un ordre différent, « impliqué » ou « emplié » ${ }^{30}$.

\section{CARACTERISATION D'UN «MONDE QUANTIQUE»}

\section{La pensée physique des phénomènes et des systèmes quantiques}

Lorsque les physiciens étudient les propriétés des systèmes qui sont à l'origine des phénomènes quantiques, avant de les ramener aux caractéristiques des dispositifs expérimentaux par lesquels ces phénomènes sont mis en évidence, ils travaillent dans le système conceptuel de la théorie quantique elle-même, avec ses grandeurs exprimées mathématiquement et leurs relations propres. Cela est particulièrement évident lorsqu'il s'agit de travail théorique à proprement parler, mais cela se marque aussi au niveau de l'étude «phénoménologique» de cette physique même, tant théorique qu'expérimentale. Il s'agit de comprendre tel ou tel processus de transformation ou d'interaction de particules et de champs (quantiques). Du point de vue de ce travail et de cette pensée théorique, le raccord avec le monde phénoménal est assuré par le fait que, pour un processus donné, les calculs théoriques sur les quantités telles que les opérateurs d'interaction et les vecteurs d'état donneront, par exemple, les amplitudes de transition entre les états initial et final qui fourniront, en définitive, combinées au volume d'espace de phases disponible ${ }^{31}$, des quantités telles que des sections efficaces d'interaction ou des durées de vie de désintégration (c'est-à-dire des fréquences statistiques) directement mesurables expérimentalement. Mais, si ce sont ces fréquences que l'on détermine effectivement par la mesure, l'objet de la pensée physique sur lequel elles nous informent reste bien l'état quantique désigné par la représentation théorique, communément appelée «le formalisme», bien qu'il s'agisse de théorie physique : c'est-à-dire telle «particule quantique», avec sa charge, son spin orienté (polarisé), ses autres «nombres quantiques»....

\footnotetext{
${ }^{29}$ Albert Einstein, Lettre à Hans S. Joachim (14.8.1954), in Einstein [1989-1993], vol. 1, p.256257.

${ }^{30}$ Bohm [1980], chapitres 5, 6, 7. Voir aussi Bohm \& Hiley [1993].

${ }^{31}$ Plus précisément, le module de l'amplitude de transition entre un état initial et un état final, élevé au carré et intégré sur le volume d'espace de phases disponible des variables, fournit la probabilité du processus considéré. Sur la théorie quantique des champs, voir, p. ex., Itzykson \& Zuber [1980].
} 
Tous ces raisonnements portent donc fondamentalement sur les grandeurs du «formalisme» de la théorie quantique, considérées de fait comme significatives du point de vue physique, et fonctionnant comme les véritables éléments de la pensée physique, de la théorie à l'expérience. On représente, par exemple, un électron et un neutrino par leurs spineurs respectifs, qui sont les fonctions d'état de ces particules quantiques à spin demi-entier, régies par le formalisme de l'équation de Dirac et celui de la théorie quantique des champs ${ }^{32}$. En les associant adéquatement aux fonctions d'état d'un neutron et d'un proton, libres ou liés dans un noyau atomique, la théorie fournit ainsi les propriétés de la désintégration $\beta$ du neutron ou, inversement, de l'interaction (faible) d'un neutrino sur un neutron, donnant un proton et un électron dans l'état final. En calculant un tel processus, les physiciens raisonnent comme s'ils le pensaient directement, et ils le font avec d'autant plus de bonne conscience que les expériences leur révèlent bien ces particules, identifiées dans les détecteurs, et qu'elles leur confirment (ou non) leurs prédictions théoriques sur le comportement de ces réactions (leur structure).

Certes, les expériences ne leur donnent accès qu'à des traces indirectes dans les détecteurs (grains d'argent révélés d'émulsion photographique, gouttelettes de liquide ou bulles gazeuses, étincelles, signaux électriques,...) qui, en dernier ressort, sont des effets macroscopiques relevant de la physique classique. Mais les règles de la physique quantique leur permettent de passer de l'un à l'autre, d'effectuer, pour ainsi dire, le «transit» entre les deux mondes : celui de leur représentation des systèmes quantiques considérés en eux-mêmes, et celui du monde macroscopique, de la physique classique des dispositifs expérimentaux. En absence d'interactions avec un environnement matériel diffus, idéalement dans le vide, des particules quantiques gardent au long de leur parcours leur caractère quantique (exprimé, pour l'essentiel, par le principe de superposition linéaire des fonctions d'état, c'est-à-dire la cohérence des phases de ces dernières), et elles le maintiennent encore lorsqu'elles entrent en collision avec une autre : toute la physique des particules et des champs quantiques est fondée sur cette idée.

Ce sont ces phénomènes se produisant entre des systèmes quantiques, quand ils sont livrés à eux-mêmes, et non pas projetés ou réduits sur des caractères ou des concepts classiques (onde, corpuscule; position, impulsion; etc.), exprimant leur «dualité » et passibles des "relations d'incertitude» ou inégalités de Heisenberg, qui constituent ce que l'on est bien en droit, finalement, d'appeler le «domaine quantique» ou le «monde quantique». Pour les physiciens qui sont habitués à travailler sur lui, ce monde est aussi réel que le domaine ou le monde classique (c'est-à-dire des objets décrits par les moyens conceptuels et

\footnotetext{
${ }^{32}$ Les matrices $\gamma_{\mu}$ de l'équation de Dirac jouent un rôle fondamental : sur elles sont construits les opérateurs d'interaction des champs des interactions électromagnétique, faible et forte. Sur les théories de ces interactions, voir Leite Lopes \& Escoubès [1995].
} 
théoriques de la physique classique). Il peut être tout aussi bien pensé que l'autre, mais seulement au moyen des concepts et de la théorie quantique, et de la manière la plus précise. Simplement, sa relation aux impressions sensibles, sources initiales de nos connaissances, est plus indirecte, et réclame davantage de médiations.

Telle est, du moins, la manière dont les physiciens d'aujour'hui auront tendance à répondre spontanément, si on les interroge sur ce que sont les « objets » sur lesquels ils travaillent. Ils rapporteront, par exemple, les résultats obtenus en détectant les neutrinos en provenance du Soleil, en termes d'oscillations des neutrinos, durant leur propagation, de l'état initial d'un certain type physique (neutrino électronique, muonique, tauique) aux deux autres ${ }^{33}$. La connaissance de ce «monde quantique» présente des particularités qui ont longtemps fait problème et suscité des interprétations diverses. Nous ne nous appesantirons pas ici sur ces questions. Nous indiquerons simplement que l'acceptation « pratique » de ce monde, fondée à la fois sur la pensée théorique et sur les résultats d'expérience, doit s'accompagner d'une élucidation conceptuelle, épistémologique, de son mode de représentation. Cette élucidation n'est pas toujours acquise, et des ambiguïtés demeurent souvent quant au statut de réalité physique de ce «monde» et au caractère pleinement physique de sa représentation.

Pour prendre un exemple, les cosmologues quantiques se trouvent, me semble-t-il, dans la nécessité de résoudre la contradiction, dont ils ne se rendent pas tous compte, entre les deux attitudes ou positions suivantes qu'il leur arrive de maintenir ensemble ${ }^{34}$. D'une part, ils professent une conception observationaliste et subjectiviste de la physique quantique, mettant en avant l'observation comme référent supposé de la théorie et le prétendu "principe » de réduction de la mesure. D'autre part, ils paraissent souscrire à une conception objective et réaliste fondamentale de cette même physique quantique dès qu'il est question de la faire servir à la considération de l'Univers dans son ensemble. Il en va à peu près de même pour bien des physiciens qui font référence à la complémentarité au sens de Bohr alors même qu'ils viennent de montrer qu'il est possible de penser physiquement des systèmes individuels, ou des états quantiques de superposition se propageant, par le fait même qu'ils peuvent les produire.

Cette question est liée à celle de savoir si la définition des systèmes quantiques peut s'affranchir de la référence à l'observation. Est-il possible de

\footnotetext{
${ }^{33}$ Les neutrinos du type $v_{e}$, produits dans les réactions nucléaires du Soleil, se transforment en partie (semble-t-il) en $v_{\mu}$ ou $v_{\imath}$ ce qui est possible par le principe de superposition (voir, p. ex., Paty [1995], et les résultats rapportés récemment (en 2000 et en 2001) dans les publications spécialisées. Voir aussi, sur les développements récents de la physique subatomique, Bimbot et Paty [1996]).

${ }^{34}$ Par exemple, tels d'entre eux, à ce colloque même.
} 
penser «directement» les systèmes quantiques, indépendamment de leurs conditions d'observation? Cette revendication de la pensée théorique apparaît à vrai dire co-naturelle au développement d'une véritable pensée de ces systèmes et de plus en plus nombreux sont effectivement les physiciens qui revendiquent que les systèmes quantiques soient pensés «tels qu'en eux-mêmes »: par exemple comme des «quantons » et non plus comme des ondes ou des particules ${ }^{35}$; que leurs attributs soient des propriétés d'existence et non plus des attributs d'observation, des «be-ables » et non plus des « observ-ables », selon l'expression de John Bell ${ }^{36}$; que l'on apprenne à voir la théorie comme étant «proprement quantique $»^{37}$. Bien des théoriciens effectuent leurs calculs en théorie quantique dans l'idée qu'ils traitent des éléments fondamentaux de la matière dont les autres sont constitués, et que ces éléments sont objectifs et réels. Certains cherchent à le justifier par des modèles physiques particuliers à partir des champs fondamentaux d'interaction. D'autres l'affirment simplement, mais il y manque cependant encore une justification épistémologique, dont ils se passent au nom des succès de la théorie quantique et de la puissance des mathématiques (ce qui peut être très pragmatique, ou au contraire très platonisant). Du point de vue de la justification physique d'une "aséité » des systèmes quantiques et de leur monde, il faut mentionner les essais significatifs de formuler une théorie quantique sans réduction par la mesure, donc en se libérant du référent contraignant de l'observation $^{38}$. Reste encore la justification par l'analyse épistémologique des notions.

L'exemple des discussions évoquées plus haut sur la distinction entre l'espace perceptif et l'espace géométrique (construit par l'entendement) peut être ici utile pour nous suggérer une marche à suivre, vers la conception d'un système relationnel de grandeurs différent, ayant pris ses distances par rapport aux données immédiates de la perception et de l'observation, et conçu en connaissance de cause par le seul entendement (grâce au rôle exemplaire de la mathématique, maîtresse en rationalité). On peut aussi considérer, de manière plus spécifique, que la spatialité concernée par la physique quantique peut être reconstruite et pensée, rendue intelligible, par l'entendement, au-delà de la perception.

En fait, la clarification est possible en considérant la théorie quantique

\footnotetext{
35 Mario Bunge parle de "quantons" pour désigner des systèmes proprement quantiques, sans réduction classique à une dualité de l'onde ou du corpuscule (Bunge [1973]).

${ }^{36}$ Bell [1987].

${ }^{37}$ Jean-Marc Lévy-Leblond s'est efforcé de penser ainsi la théorie quantique, en commençant par «nettoyer» ses pseudo-concepts en faisant pleinement droit à leur nature de quantons, et en modifiant en conséquence la manière d'enseigner la "Quantique" (Lévy-Leblond [1977a, b, 1988], Balibar et Lévy-Leblond [198?]).

${ }^{38}$ Parmi les premiers essais dans ce sens, mentionnons la théorie de David Bohm déjà citée, de 1952, et la "conception de l'état relatif" d'Hugh Everett III, de 1957. Voir Ben Dov [1989?], Freire [1995].
} 
telle qu'elle est, et en effectuant une réflexion épistémologique sur les grandeurs théoriques utilisées en physique quantique (avec comme base les concepts et les relations de la mécanique quantique) et sur leur signification du point de vue physique. En l'absence d'une telle élucidation, l'attitude courante reste encore très ambigüe quant à la signification du rapport de la théorie à l'expérience ; l'on évite en général toute discussion en rapportant les grandeurs théoriques au « formalisme » et à ses règles d'interprétation. Et, pour ces dernières, on emploie encore bien souvent le langage de l'observation, héritage de l'« interprétation orthodoxe » ou « philosophie de la complémentarité ».

La question de fond apparaît bien être simplement celle-ci : les grandeurs du «formalisme quantique » sont-elles des grandeurs mathématiques, ou des grandeurs physiques, mathématisées, comme on le considère pour les autres théories physiques? Admettre la deuxième réponse (que ce sont des grandeurs physiques) permet de constater un déplacement du problème de l'interprétation. Au lieu d'être un problème sur la nature de la connaissance (observationalisme contre réalisme) il s'agit désormais, de manière plus banale, de garantir le caractère physique approprié d'une grandeur formulée mathématiquement.

L'intelligibilité du «monde quantique» se trouve grandement simplifiée si l'on adopte le point de vue selon lequel il est légitime de parler d'un « domaine quantique » propre. Elle demande, pour cela, un examen des divers aspects de la représentation théorique, concordants avec la connaissance expérimentale des phénomènes, et des problèmes d'interprétation qui leur sont reliés. Mentionnons-les ici pour mémoire (nous avons étudié certains d'entre eux par ailleurs ${ }^{39}$ ) : le concept d'état et de fonction, ou vecteur, d'état et les grandeurs dynamiques appelées « observables »; la non-séparabilité locale ; le rapport entre état quantique et probabilités et la double signification de ces dernières (théorique et statistique); le rapport entre les domaines quantique et classique ; la pensée théorique de la physique emmenée par les formes mathématiques, pour aboutir à une représentation légitimement physique ; le problème de l'espace physique, vu du monde quantique (objet du présent exposé); la décohérence et la mesure ; puis le problème de la gravitation quantique et de la matière de la cosmologie.

Nous considérons, dans ces analyses, que l'on doit admettre comme physique, parlant de concepts ou de représentation, ce qui est théoriquement significatif en considération des phénomènes décrits. En ce sens, des grandeurs physiques exprimées mathématiquement sont légitimées par l'intelligibilité qu'elles procurent dans un domaine physique donné par l'expérience des phénomènes. Il convient donc de concevoir une extension de sens de la notion de

\footnotetext{
${ }^{39}$ Pour une discussion plus détaillée, dans cette perspective, des questions d'«interprétation physique», voir (respectivement) : Paty [2000c, à paraitre, c ; 1999 ; à paraître, b ; 1988, chap. 6, 1986 et $1989 ; 2001 \mathrm{a} ; 2000 \mathrm{~d} ; 2002 ; 2000 \mathrm{~b}]$.
} 
grandeur physique, au-delà des limitations généralement admises pour cette notion aux seules grandeurs «à valeurs numériques » et "directement mesurables ». On admettra, dès lors, que la fonction d'état (ou «fonction d'onde »), définie comme vecteur dans un espace de Hilbert, soumise au principe de superposition, et les variables dynamiques correspondantes (les « observables ») décrites sous forme d'opérateurs agissant sur la première, sont des grandeurs physiques décrivant le système quantique. Ces grandeurs sont les grandeurs appropriées à une telle description, au contraire des grandeurs classiques correspondantes (quand il y en a), qui n'en décrivent que des projections sur un système classique de grandeurs.

En, fait, pour le dire d'un mot, les termes du débat quantique traditionnel apparaissent archaïques aujourd'hui, si l'on veut bien prêter attention à la connaissance effective des systèmes quantiques dont on dispose, qui va très audelà de ce qu'autorisait la conception orthodoxe "de la complémentarité ». Les connaissances récentes acquises sur des systèmes quantiques individuels, en particulier, montrent comment la théorie quantique implique et décrit directement de tels systèmes. De même, dans l'autre sens, les systèmes collectifs à grand nombre de composants identiques indiscernables, tels que la condensation de Bose-Einstein, récemment observée, qui paraissaient naguère une vue de l'esprit (et l'indiscernabilité, un défaut de connaissance), sont démontrés exister effectivement, objectivement, dans la nature, remplissant exactement les traits que la théorie leur prévoyait.

La signification philosophique d'une extension de sens de la notion de "grandeur physique»

L'extension de sens indiquée pourrait simplifier considérablement notre compréhension des domaines de connaissance correspondants, et cette simplification serait même radicale en ce qui concerne les problèmes d'interprétation. Elle rétablirait, comme conséquence immédiate, la signification et l'usage de la notion d'objets d'une théorie conçue comme description et représentation. Le genre de réalisme que nous considérons ici est, certes, un réalisme critique, celui de constructions symboliques pour représenter la "réalité », et conçu comme un programme pour l'élaboration scientifique ${ }^{40}$.

Prenons les concepts d'état et de grandeur quantiques. Etant donné que la théorie quantique permet d'expliquer tant de groupes de phénomènes et d'en donner des modèles puissants, il est tentant de la concevoir comme une théorie fondamentale d'un monde d'objets. Et telle est bien, de fait, comme nous l'avons vu, la manière spontanée dont les physiciens la pratiquent, bien qu'ils se heurtent à des difficultés quand (mais seulement alors) ils en viennent à s'interroger sur la transition de ce domaine quantique au domaine classique des

40 Paty [1988], en particulier les chapitres 1 et 10 ; [1993], chapitre 9. 
appareils de mesure. L'interprétation «courante » («de Copenhague ») professe qu'il n'existe pas, scientifiquement, de signifié conceptuel désigné dans la théorie, pouvant être considéré comme un objet (c'est-à-dire une entité ayant des propriétés) et que ce signifié conceptuel (ce qu'on appelle «objet» de la théorie, c'est-à-dire l'état du système) n'existe qu'en relation à des conditions données (et optionnelles) de préparation pour la mesure.

Mais, en réalité, les scientifiques qui travaillent avec des objets physiques quantiques récusent par leur pratique (même s'ils ne l'osent pas de façon explicite concernant des questions de "philosophie») cette interprétation courante, en élaborant une nouvelle objectivité conçue sur le mode de l'ancienne, mais faisant appel à des concepts et à des grandeurs dotées d'un sens plus large que les grandeurs classiques. Il est vrai qu'un état quantique n'est accessible à l'expérience qu'indirectement, mais ceci n'affecte pas la possibilité d'en avoir connaissance. La modification épistémologique essentielle aura consisté, en vérité, en une extension de sens (restée implicite) du concept de grandeur ou de quantité physique (en particulier, celui d'état physique), à des entités qui ne sont pas simplement à valeur numérique. Cette extension est légitimée par les phénomènes, dans une acception de ce terme qui ne les réduit pas à leur appréhension par la perception, mais qui les conçoit selon l'entendement, c'est-àdire selon leur capacité à être portés à notre connaissance, et elle est réalisée, pour peu qu'on y réfléchisse, dans la pratique sinon dans la conscience qui en est prise, par l'essentiel du « formalisme » même de la théorie quantique.

Cette extension a été, de fait, préparée par les travaux des physiciens théoriciens de la physique quantique sensibles aux propriétés formelles, mathématiques, de la théorie, comme Max Born, Werner Heisenberg, Paul Adrian Dirac, John von Neumann, Hermann Weyl et d'autres, où les grandeurs physiques classiques étaient remplacées par des "grandeurs quantiques » différentes d'elles en premier lieu par leurs propriétés formelles (superposition pour les fonctions d'état, non-commutation pour les opérateurs,...). Par exemple, les nombres- $q$, non commutatifs, proposés par Dirac pour remplacer les nombres-c ordinaires, suggèreraient immédiatement une extension de sens comme celle que nous venons d'indiquer ${ }^{41}$. Mais ces pionniers n'avaient cependant pas cru devoir proposer d'emblée ces constructions formelles comme de simples extensions de sens des grandeurs physiques, parce que les questions d'interprétation alors soulevées ne paraissaient pas les y autoriser. De telles grandeurs restaient seulement mathématiques, leur rapport aux phénomènes physiques étant réglé par l'« interprétation ». La pierre d'achoppement était alors essentiellement le passage du classique au quantique, avec la question de la «mesure » au sens quantique ${ }^{42}$.

41 Voir les travaux de Dirac de 1926. Cf. Mehra \& Rechenberg [1982], vol. 4, p. 162 et suiv., Darrigol [1992].

42 Paty [1999a] ; [à paraître, b]. Sur la formalisation de la mécanique quantique et l'introduction des grandeurs mathématiques abstraites, voir, en particulier Dirac [1930], von Neumann [1932], 
Considérer comme grandeur physique au sens plein du terme des vecteurs d'état quantiques dans la forme de superpositions linéaires et des « opérateurs-observables » ne commutant pas nécessairement, pourvus de valeurs propres affectées de probabilités, cela implique de renoncer à la connexion étroite, voire à l'identification, de propriétés avec ce qui est ou pourrait être mesuré au sens classique et numérique, et de concevoir différemment ce qu'est une propriété, relationnelle, en la rapportant au système ou à l'état tel qu'il est construit intellectuellement par un processus d'abstraction et d'élaboration théorique intégrant des données factuelles. Des propriétés conçues de la sorte ne sont plus contextuelles et peuvent être dites intrinsèques : telles sont, dans cette perspective, les propriétés des «particules » quantiques élémentaires (photon, électron, quark, etc....), munies de leur charge, de leur spin, etc., et des champs quantiques engendrés par des transformations de symétrie.

Ces propriétés ne dépendent aucunement des circonstances de leur observation, mais elles sont reconstituées à partir d'observations expérimentales fournissant des valeurs de grandeurs correspondant à des propriétés contextuelles, affectées de probabilités mesurées par des fréquences d'événements. A cet égard, les probabilités, loin de constituer une limitation de la connaissance, permettent la détermination des grandeurs intrinsèques et de leurs relations, qui sont celles dont la théorie se soucie principalement, à partir de la distribution spectrale de leurs composantes.

Weyl [1928], etc. Sur les diverses manière d'aborder le problème de la mesure en mécanique quantique, d'abord posé par von Neumann [1932], voir Wheeler \& Zurek [1983]. 


\section{4. \\ LA PHYSIQUE QUANTIQUE ET LE CONCEPT “CLASSIQUE” D’ESPACE.}

\section{Les limitations du concept d'espace pour la physique quantique}

Nous ne passerons pas ici en revue de manière détaillée les différentes limitations que la théorie quantique oblige à apporter au concept d'espace : probabilité de présence, et non pas localisation certaine; absence de trajectoire définie; inégalités de Heisenberg entre la position et l'impulsion; caractère étendu, c'est-à-dire non local, d'un système quantique capable d'interférer avec luimême dans un dispositif de diffraction; indiscernabilité des identiques qui gouverne des comportements collectifs comme s'il s'agissait d'«influences à distance»; non localité et corrélations quantiques à distance (ou non séparabilité locale des systèmes quantiques $)^{43} \ldots$. Ces caractéristiques n'étaient dues, selon l'interprétation orthodoxe, qu'à l'impossibilité de s'affranchir, pour les phénomènes quantiques, de la référence à l'observation et de considérer des systèmes quantiques en eux-mêmes ${ }^{44}$. Pourtant, si l'on admet la signification, du point de vue de la pensée physique (et de la réalité physique correspondante décrite par la première), d'un monde autoconsistant de propriétés et de systèmes quantiques, ces limitations doivent être conçues comme des caractères objectifs. Elles signifient, d'une part, que le concept d'espace de la géométrie et de la physique classiquerelativiste ne peut être utilisé tel quel comme concept de la physique quantique et, d'autre part, que des systèmes quantiques venant à occuper une région d'espace donnée, précisément définie ou macroscopique, confèrent (tant qu'ils conservent ces propriétés, en cohérence de phase) à l'espace occupé des propriétés «quantiques», différentes des caractères habituellement attachés à un tel espace, tels, notamment, que sa divisibilité en points élémentaires.

Les grandeurs classiques qui servaient d'éléments de base à la théorie physique classique et ont permis d'élaborer sa formulation lagrangiennehamiltonienne ont perdu, avec la physique quantique, leur signification physique directe. Si la théorie quantique a gardé, dans sa construction, la part «formelle » (la formulation hamiltonienne), ce fut à la condition d'abandonner la signification, et même la forme, des grandeurs initiales. A part le temps, qui n'a pas d'équivalent comme opérateur, les autres variables ont été remplacées, dans l'« analogie formelle » qui a gouverné la formulation de la mécanique quantique, par des grandeurs dynamiques sous forme d'opérateurs (les «observables»). Les équivalents, ou plutôt les correspondants (éventuels), des variables classiques, fournis par la théorie quantique, sont les valeurs propres de ces opérateurs pour un

\footnotetext{
${ }^{43}$ Sur la non séparabilité, voir Bell [1987], Aspect [1983], ...

${ }^{44}$ Sur l'interprétation "orthodoxe" ou "de Copenhague", voir notamment : Bohr [1958], Rosenfeld [1979].
} 
état propre solution de l'équation d'état (et soumis au principe de superposition, de telle façon que toute superposition linéaire d'états propres est aussi un état possible du système). Ainsi un système quantique n'a-t-il une position dans l'espace que sous les conditions qu'octroie le statut de valeur propre. La position spatiale d'un système quantique n'est attribuable que de manière contextuelle, et soumise ainsi aux limitations et conditions rappelées plus haut. Elle ne fait pas partie des concepts «propres» des systèmes quantiques, et l'on doit, pour la considérer, préciser à quelles circonstances relatives elle est conditionnée. L'absence de trajectoire déterminée, la non-localité, l'extension spatiale, des systèmes quantiques résultent de cette caractéristique.

\section{Engendrement d'espace, cosmogénèse, et espace vide de la propagation des sytèmes quantiques}

Lorsqu'on parle de systèmes quantiques tel que les décrit la théorie quantique, on est amené à considérer que leurs "propriétés », au sens plein du terme (c'est-à-dire ce qui les caractérise en propre, indépendamment de tout «contexte» au sens expérimental du terme), sont autodéfinies par cette théorie. Mais, en même temps, on les considère en fait le plus souvent dans le cadre d'un espace déjà donné, qui est celui, admis antérieurement, de notre expérience (de l'ensemble de nos expériences), du monde environnant et du cosmos. C'est l'espace (-temps) classique, de la physique relativiste (restreinte et générale) et de la cosmologie : celui où ces systèmes quantiques se propagent et celui où ils s'agrègent ensemble pour former des atomes et des molécules et des systèmes de telles entités, qui, au niveau macroscopique, apparaissent très bien définis spatialement.

Dans le premier cas, celui de la propagation, l'espace classique (celui de la distance parcourue, par exemple par des particules d'accélérateurs, ou des neutrinos en provenance du Soleil) est pris, avec le temps, comme l'une des variables suivant lesquelles on détermine l'évolution du système, bien qu'elle ne soit pas inhérente à ce dernier : du moins correspond-elle à une grandeur physique issue de l'expérience, mais qui reste extérieure à la définition du système quantique.

Dans le second cas, l'espace n'est pas donné à l'avance, et le volume d'occupation se détermine en fonction de l'agrégation des constituants : il est d'autant moins défini que le nombre de constituants quantiques est petit. A cet égard la pensée des systèmes quantiques et de leur agrégation en "grands" systèmes qui occupent un espace défini paraît assez semblable à la constitution de l'espace dans une cosmogénèse, sans espace préexistant, l'espace se créant au fur et à mesure du déploiement de l'Univers. A ceci près, cependant, que la cosmologie quantique suppose toute la matière concentrée, sans espace ou quasiment, avant son déploiement dans l'espace, la notion qui est ici en cause 
étant celle d'espace comme contenant. L'espace de l'Univers s'engendre avec le déploiement (et la dilution d'énergie) de la matière. La cosmogénèse quantique (et ce trait appartient aussi bien à la relativité générale) correspond à l'engendrement de l'espace (et de l'espace-temps) par la matière. Selon la cosmologie quantique (qui n'est encore qu'une série de modèles théoriques, rassemblés dans le «modèle standard $»)$, cette matière initiale est purement quantique, et cet engendrement de l'espace et du temps se fait par différenciation des états physiques ${ }^{45}$ et découplage progressif des interactions, sauf l'interaction gravitationnelle.

$\mathrm{Au}$ début du processus se situe l'état de «gravitation quantique », sur lequel nous n'avons aucune certitude ${ }^{46}$. En considérant l'état actuel de l'Univers, l'espace physique (et le temps), issus de cette genèse, nous sont donnés, et les systèmes quantiques élémentaires s'y propagent, et parfois s'y agrègent en se localisant. On doit constater cet état de chose, sans bien savoir comment raccorder cette coexistence de deux types d'«objets physiques» aussi différents que des systèmes quantiques, pour lesquels le concept d'espace reste étranger à leur définition, et l'espace vide environnant, dans lequel ils «existent». On doit, en tout cas, considérer ici deux réalités physiques: les systèmes quantiques, et l'espace physique, créé dans la genèse cosmique, dans lequel ils se trouvent. A quoi s'ajoute que cet espace vide n'est pas vraiment vide, conceptuellement et physiquement, puisqu'il comprend le vide quantique, qui est matériel, représenté en termes de champs quantiques (et de particules virtuelles, corollaires de ces champs), en sorte que les deux entités (systèmes quantiques, espace physique) s'appellent l'une l'autre, la primauté conceptuelle revenant, du point de vue adopté, aux concepts quantiques (et, fondamentalement, à celui de champ quantique).

Il faudrait analyser davantage le rapport de ces deux «régimes», ou de ces deux représentations du monde physique, les systèmes quantiques et l'espace physique. Que peut-on dire d'un système quantique qui se propage ? Il ne se localise que lorsqu'il interagit avec le monde de l'espace-matière classique, par exemple : l'impact sur l'écran dans une expérience d'interférence ; la localisation par détection d'une particule d'un faisceau; le confinement par champ magnétique, électrique, gravitationnel. Laissons provisoirement la question en suspens.

\section{L'espace et les systèmes quantiques individuels}

La réponse traditionnelle de l'interprétation « complémentariste » de la mécanique quantique pour maintenir à la fois l'espace classique et la spécificité quantique faisait appel à une dualité, ou une complémentarité, entre une représentation en termes d'invariance et de conservation et une représentation spatio-temporelle, qui se justifiait en invoquant le caractère statistique des

\footnotetext{
${ }^{45}$ Paty [2000b].

46 Sur les problèmes d'une théorie quantique de la gravitation, voir, p. ex., Kouneiher [1998].
} 
prédictions de la théorie quantique. Ce dernier résultait du rôle fondamental, de principe et référentiel, de l'observation. Cependant, la conception de la connaissance non seulement par, mais selon l'observation, apparaît de plus en plus comme une conception ad hoc, qui se satisfait d'une demi-connaissance, et dont l'insuffisance se révèle avec celle d'une conception ambiguë de la signification de résultats probabilistes, la «solution statistique», dont elle est solidaire ${ }^{47}$. L'insuffisance de la «solution statistique » est avérée aujourd'hui par les faits eux-mêmes. Il est, en effet, clair désormais que la théorie quantique (par la fonction d'état, les grandeurs dynamiques et les équations d'état) peut caractériser des systèmes individuels qui correspondent bien à des entités physiques («particule» unique, atome unique, photon unique).

Un certain nombre de physiciens quantiques de la mouvance « orthodoxe» avaient bien conscience, en fait, de la possibilité de rapporter les propriétés quantiques à des systèmes physiques individuels. Mais ils s'interdisaient de concevoir de tels systèmes indépendamment de leurs conditions d'observation ; il faudrait, pensaient-ils, pour s'assurer du caractère individuel d'un système quantique, le compter et, pour cela, le détecter (par interaction) suivant son aspect corpusculaire, donc détruire son aspect ondulatoire dual et, partant, son caractère quantique. De toute façon, la conception " orthodoxe» au sens strict n'envisageait l'éventualité d'un système quantique individuel que si celui-ci était dûment « chaussé », pour ainsi dire, de l'instrument pour le détecter, et celui-ci le liait indissolublement à l'espace physique au sens classique-relativiste. Le problème d'une éventuelle difficulté à penser l'espace pour les systèmes quantiques ne se posait donc pas, puisque ces systèmes ne pouvaient être conçus qu'immergés dans cet espace, cadre de l'observation et donc des phénomènes rapportés à des «systèmes-en-observation», seuls concevables. L'espace connaissait seulement, en raison des nécessités de l'observation particulière aux phénomènes quantiques (gouvernée par l'indivisibilité du quantum d'action), des limites d'utilisation, dont nous avons parlé (fondamentalement, celle des relations d'inégalité de Heisenberg).

Cette vue, soit dit en passant, nous fait comprendre pourquoi la réponse de Bohr à l'argument EPR (d'Einstein, Podolsky et Rosen) aurait été à elle seule strictement incapable de formuler clairement l'inséparabilité quantique comme concept et propriété ${ }^{48}$. Celle-ci fut, en fait, énoncée par Einstein, en négatif, comme difficulté d'une séparabilité locale pour des systèmes individuels, précisément parce qu'il avait pensé le problème de la représentation de tels systèmes (ce qu'il appelait des «états physiques réels ») ${ }^{49}$. En bref, avec la conception «observationaliste » orthodoxe, il était inutile, hors de question et,

\footnotetext{
${ }^{47}$ Voir Bohr, Born, Pauli...

${ }^{48}$ Einstein, Podolsky \& Rosen [1935], Bohr [1935].

${ }^{49}$ Voir, notamment : Einstein [1948]. Sur les développements et la signification de cette question,
} 
somme toute, impensable, de pousser plus avant la pensée critique de l'espace à propos des systèmes quantiques.

En ce qui concerne les systèmes quantiques individuels, la réalisation, par construction, de faisceaux où les particules quantiques sont « égrenées " une à une, avec une résolution temporelle significative contrôlable, permet, de nos jours, de faire des expériences avec des particules quantiques individuelles, et implique donc, même pour les physiciens (ou les épistémologues) observationalistes les plus invétérés, la nécessité d'une pensée physique de ces systèmes. A la caractérisation théorique d'individualité pour des systèmes quantiques, taxée naguère de purement formelle et sans portée physique, correspond désormais le donné factuel : la possibilité d'étudier effectivement des «particules quantiques » individuelles, qui était niée dans une perspective strictement opérationaliste, est désormais acquise comme un fait d'expérience ${ }^{50}$. C'est donc bien pour de tels systèmes quantiques individuels, auxquels on doit désormais rapporter la description théorique quantique, que le concept habituel d'espace apparait comme limité, et donc comme insuffisant.

Mais qu'est-ce que l'espace physique pour les systèmes quantiques ? Les difficultés épistémologiques suscitées par le concept «classique » d'espace physique nous invitent à ne pas mettre ce dernier directement en avant. Il n'y a pas d'«espace physique » qui nous soit donné indépendamment des phénomènes. Autrement dit, pour les phénomènes quantiques, nous admettrons au mieux qu'il puisse y avoir un « espace physique quantique », différent de l' "espace physique classique » (qui inclut celui au sens de la théorie de la relativité).

Il nous faut donc considérer, pour ce qui est des phénomènes quantiques, le concept et la grandeur espace sous deux aspects : celui d'un espace physique dans un sens quantique, et celui du rapport d'un tel espace à l'espace physique classique. Concernant le premier : que nous dit la physique quantique, considérée comme physique d'un «monde quantique» (ou «monde des phénomènes quantiques »), sur l'espace physique; autrement dit: la physique quantique définit-elle (ou caractérise-t-elle) un "espace quantique » ayant des caractéristiques particulières ? Concernant le second aspect : comment s'effectue le raccord entre l'espace selon le monde quantique et l'espace du monde macroscopique, ou espace physique classique?

A la première question, la théorie quantique offre une réponse, par la manière même dont elle s'est constituée. A partir de la notion première de groupe de transformation et d'invariance sont formés les opérateurs, à l'aide de générateurs infinitésimaux : c'est la manière minimale dont intervient la notion de

\footnotetext{
voir Paty [1986, 1988, 1995, et à paraître, a].

${ }^{50}$ Voir, entre autres, Grangier [1986]. Sur la notion d'état quantique, sa description théorique et sa contrepartie physique, telle qu'on peut la caractériser aujourd'hui, voir Paty [à paraître, b].
} 
spatialité dans la formation des grandeurs quantiques et elle concerne la grandeur désignée par l'opérateur de position. A la seconde interrogation, la réponse traditionnelle (mais insuffisante au regard de notre attente sur les systèmes quantiques), est celle de la mesure. Mais il faut considérer aussi l'espace physique (au sens classique) de la propagation d'un système quantique, dont nous avons parlé plus haut, et pour lequel nous avons souligné la coexistence (ou dualité de fait) entre les systèmes quantiques et l'espace physique classique dans lequel ils se propagent. On doit aussi considérer la possibilité, déjà brièvement mentionnée, d'un espace émergent à partir de certains types d'agrégats de systèmes quantiques, engendrant un certain volume d'espace qu'ils occupent; nous y revenons pour terminer.

5.

\section{PRE-ESPACE PHYSIQUE QUANTIQUE ET ESPACE PHYSIQUE CLASSIQUE.}

Les changements de concepts et de représentations, tels que ceux qui ont abouti à la formulation de la théorie quantique, s'ils ont été rendus nécessaires par l'expérience et les moyens "classiques» de celle-ci, ont été effectués au niveau de l'entendement, par le jeu des objets de pensée et des opérations sur eux, permis par la puissance des propriétés relationnelles de grandeurs mathématiques. Leur adéquation aux problèmes physiques considérés leur donne valeur de représentation physique effective. Ces transformations dans les structures et les soubassements mêmes de la connaissance en physique (et non pas seulement dans ses contenus) correspondent à des élargissements successifs des éléments de rationalité dans la pensée, qui permettent d'assimiler notre expérience des données, tant des formes (objets formels) que du monde de l'empirie. Ainsi la physique a-t-elle pu s'abstraire de l'espace physique classique pour penser un «monde quantique » qui n'est plus organisé autour de cette notion, selon un processus exemplaire où, tout en entrant à un certain titre dans la construction des grandeurs quantiques, l'espace y subit une mutation de sens. Cette mutation n'est pas de l'indifférence, car les systèmes quantiques ont très souvent à voir avec l'espace physique, selon ce que nous avons entrevu. Nous allons considérer, pour terminer, un cas particulier de ces rapports, lorsque des systèmes quantiques en parfaite cohérence de phase s'agglomèrent pour constituer une certaine région de l'espace physique, tout en étant totalement quantiques.

Une expérience astronomique par la pensée : existe-t-il des étoiles «condensats de Bose-Einstein»?

Comment pouvons-nous nous représenter l'espace de systèmes quantiques se trouvant en cohérence de phase ? Considérons le cas d'un grand nombre de particules ou systèmes quantiques identiques se trouvant agglutinés dans le même état physique: elles engendreraient à coup sûr de l'espace, 
occupant, comme on le dit pour la superfluidité ou pour la condensation de BoseEinstein, «tout l'espace à leur disposition ». Ce qui est une façon de parler, car l'espace à leur disposition, c'est l'espace qu'elles peuvent occuper en restant dans ce même état, c'est-à-dire que c'est l'espace engendré par leur agrégation, laquelle constitue un «nouvel état de la matière». On parle désormais, pour les condensats observés en laboratoire, à partir de millions d'atomes identiques «tombés» sur le même état quantique, et visibles au microscope, de leur forme (ellipsoïdale...). Quelle est la nature, et la structure, de ce volume fini d'espace occupé par un tel atome quantique multiple ? En tout cas il n'est pas contitué de points, puisque le même état occupe tout l'espace, d'une extrémité à l'autre, sans division possible. C'est un continu indivisible, inséparable, une «totalité», à des dimensions macroscopiques.

On pourrait pousser l'image plus loin encore. Imaginons une « expérience par la pensée » (ou, plutôt une «observation par la pensée », puisqu'elle porte sur l'astronomie), d'un effet de condensation de Bose-Einstein : que serait un espace de dimensions astronomiques empli de matière quantique indiscernable et cohérente telle un condensat de Bose-Einstein, sur la surface (ou dans le volume) d'une "étoile de Bose-Einstein », à supposer que de tels objets puissent exister?

Mais, tout d'abord, l'existence de tels objets est-elle possible ? L'idée m'en était venue en réfléchissant à la propriété fondamentale des systèmes quantiques qu'est l'indiscernabilité des systèmes identiques, et qui se présente de deux façons : celle des fermions et celle des bosons, avec les propriétés de symétrie statistique correspondantes (antisymétrique pour les fermions, avec pour conséquence le principe d'exclusion de Pauli ; symétrique pour les bosons, avec comme effet la condensation de Bose-Einstein de particules identiques dans un même état quantique). Le principe d'exclusion est responsable de l'organisation de la matière atomique (la classification périodique des éléments, par la répartition des électrons en états différents, dont chacun ne peut être occupé que par un seul d'entre eux), mais il détermine également la structuation d'étoiles qui sont constituées de gaz dégénérés de fermions, comme les naines blanches (électrons) et les étoiles à neutrons...

Ne pourrait-on concevoir, de manière symétrique, des objets célestes dont la structuration serait directement expliquée par la statistique de BoseEinstein, par exemple sous la forme d'un condensat, avec ou sans effondrement de l'étoile ? Ayant demandé à un astrophysicien de mes amis ce qu'il pensait d'une telle éventualité, il me répondit qu'il lui semblait, à première vue, qu'il ne pourrait «y avoir aucune région de l'Univers aussi froide que cela ». Il s'en expliquait ainsi : «Puisque le rayonnement à $3 \mathrm{~K}$ est partout présent, aucun système ne peut être inférieur à cette température (à moins qu'il n'interagisse ni avec les photons ni 
avec les neutrinos) $»^{51}$. Toutefois, cette fin de recevoir ne me satisfaisait pas. En effet, il y a désormais au moins un endroit de l'Univers, à savoir la planète Terre, où un tel froid a été réalisé, certes artificiellement, par les êtres humains, en laboratoire, et durant un laps de temps fini (et très petit). La nature livrée à ellemême devrait pouvoir faire, pour le moins, aussi bien : on peut envisager des fluctuations de température dans une région donnée de l'Univers. On pourra, certes, objecter que les interactions avec le reste du fond cosmique fossile réchauffereraient le système local, empêchant une agrégation un peu stable de grandes quantités de matière. Mais on peut aussi imaginer une production, par quelque processus naturel encore, d'une sorte de puits de basse température qui tendrait à maintenir cet agrégat dans le froid quasi absolu tandis que le réchauffement compensatoire se ferait à l'extérieur: une sorte de machine thermique naturelle, un réfrigérateur stellaire, en quelque sorte.

On pourrait encore imaginer une sorte de matière bosonique, indifférenciée, ou sous forme de paires de quarks et de gluons, dont l'état le plus bas (l'énergie du point zéro, "zero point energy») pourrait être le centre d'une condensation de Bose-Einstein. Des particules supersymétriques, à supposer qu'elles existent (ce que rien ne permet encore de dire), ne pourraient-elles s'agréger en étoiles, symétriquement à ce que font les particules ordinaires, et éventuellement, pour les bosons supersymétriques (contrepartie des fermions ordinaires, les nucléons et les électrons), se condenser sur un état d'énergie minimale par effet Bose-Einstein ? (et l'on pourrait envisager un condensat pris dans tout le volume d'un tel objet céleste). Interrogé, Pierre Fayet, dont les travaux sur les supersymétries font autorité, m'a répondu qu'une telle situation était effectivement pensable, d'autant plus que les particules supersymétriques contreparties des quarks (qui sont des fermions), à savoir les "squarks", sont des bosons, susceptibles de se condenser de la sorte : il en avait d'ailleurs eu l'idée, brièvement évoquée dans l'un de ses premiers articles sur le sujet ${ }^{52}$. L'hypothèse qu'il puisse exister dans le cosmos de nombreuses étoiles de Bose-Einstein de cette sorte ne serait donc pas si folle...

Considérons donc une région d'espace physique, par exemple, la surface d'une étoile compacte ultrafroide, aux environs de 0 Kelvins ou au plus à la «température de Fermi », dont un point de la surface constituerait un centre localisé de condensation, et des atomes bosoniques identiques (quels qu'ils soient) refroidis et portés à l'état du "point d'énergie zéro ", sur lequel ils tomberaient tous ensemble. Si cela est pensable, c'est donc possible et cela devrait exister, comme dirait Leibniz, voire cela existe déjà quelque part dans l'Univers, et il suffirait d'aller l'y observer. L'état du condensat occuperait toute l'étendue à sa disposition, peut-être toute la superficie (macroscopique) de l'étoile, l'ensemble des milliards de milliards de milliards d'atomes mobilisés, agrégés en un seul état

\footnotetext{
${ }^{51}$ Marc Lachièze-Rey, communication privée par courrier électronique (8 nov. 1999).

${ }^{52}$ Pierre Fayet, communication privée, décembre 2000. Voir Fayet [1985].
} 
quantique se trouvant très largement délocalisé (aux dimensions du volume occupé).

L'espace physique occupé par le condensat serait un espace de points en phase... (c'est-à-dire sans points) et l'on serait curieux de savoir de quoi un tel état aurait l'air, en comparaison avec un espace physique ordinaire de superficie équivalente. On peut le concevoir comme extension à un très grand domaine spatial de ce qui a été déjà observé pour la superfluidité (un fluide parfait sans viscosité remontant les parois du vase) ou, mieux encore, pour les condensats purs de Bose-Einstein d'ores et déjà produits ${ }^{53}$. Pour l'observer, les physiciens pourraient s'équiper d'un télescope «analyseur quantique» braqué sur l'espace étrange de cet objet lointain, ou encore armer un vaisseau spatial en vue d'une expédition vers ces contrées du «wilderness» quantique inviolé, pour en examiner de près les propriétés peu communes. On pourrait même imaginer en faire un film, en cette année 2001, "A quantum star space odyssey », "Une odyssée vers l'espace de l'étoile quantique $»^{54}$.

\section{Et d'autres rapports d'espace...}

Il est beaucoup plus difficile de se représenter l'espace des systèmes (à très peu de «particules ») « corrélés à distance », qui est en fait, étendu à volonté, puisque les distances de corrélations peuvent y être arbitraires. Il est clair que ces corrélations ne peuvent pas se concevoir en termes de communication ou de jonction dans l'espace, comme s'il y avait une tige rigide entre les sous-systèmes pour les corréler deux à deux, une communication instantanée se produisant entre les extrémités; ce serait revenir à l'action à distance newtonienne, et nier la relativité. En vérité, si l'on examine comment la théorie décrit de tels systèmes, on constate qu'elle ne fait tout simplement pas intervenir l'espace dans leur corrélation, et la relativité restreinte n'est pas concernée. Il faut admettre que cette corrélation, cette non-séparabilité locale, est un principe premier, ou une propriété première de ces systèmes, et qu'elle pré-existe à la considération de l'espace. Mais nous avons affaire ici à un très petit nombre de particules ou systèmes (deux, trois, pas beaucoup plus), dont la définition n'appelle pas la notion d'espace (ce qui est physiquement significatif, ce sont les variables propres de leur champ).

Prenons encore l'espace physique du vide quantique ${ }^{55}$, qui n'est « agité constamment de soubresauts virtuels » que par manière de parler, parfaitement inexacte : les images complaisantes faisant appel à des intuitions macroscopiques sont ici totalement inopérantes. C'est la théorie quantique des champs, et elle seule, qui peut nous aider à nous représenter ce vide prompt à réagir aux

\footnotetext{
53 Cornell \& Wiemann [1998]. Sur la condensation de Bose-Einstein, voir Griffin, Snoke \& Stringari [1995].

${ }^{54}$ Une version modernisée et futuriste des antiques peplums...

${ }^{55}$ Diner \& Gunzig [1998].
} 
excitations de la matière et des champs.

Considérons, enfin, l'espace de la propagation des systèmes quantiques en cohérence de phase. Le phénomène limite de la jonction de l'espace quantique avec l'espace classique est évidemment celui de la décohérence, récemment produit et observé en laboratoire ${ }^{56}$, qui se produit naturellement, par le seul jeu des systèmes physiques en présence et en interaction. L'espace classique est alors celui de l'environnement matériel, où ont lieu de nombreuses interactions successives du système avec les atomes (ions et électrons) du milieu. Alors, les caractères quantiques du système (par exemple, un état de superposition linéaire) sont progressivement, quoique très rapidement, dissous dans les innombrables interactions avec le milieu ambiant. Ce processus, naturel, est différent de celui de la mesure, qui est imposé d'une manière contraignante par les caractéristiques de l'appareillage classique (imposant ainsi une "réduction" des caractères quantiques à des caractères classiques choisis à l'avance $)^{57}$.

On retiendra cependant ce trait, qui n'était pas acquis voici peu de temps encore, qu'un état de superposition de type quantique pour un système «mésoscopique » d'atome et de champ imbriqués a été observé se propageant sur un certain parcours fini. On pourra faire remarquer que cet état de chose est admis sans autre forme de procès en physique des particules élémentaires, avec les particules quantiques électriquement neutres comme les mésons $K^{0}$ et les neutrinos, voire les neutrons, pour de grandes distances sur lesquelles ces systèmes quantiques restent isolés de toute matière environnante jusqu'à leur interaction, et qu'il constitue l'un des principes d'explication de la physique des champs quantiques de ce domaine (il s'agit des oscillations de ces systèmes) ${ }^{58}$. La différence avec la décohérence observée est, pour cette dernière, la quasi visualisation du phénomène, avec des atomes géants et des champs à la frontière du classique, phénomène qui est presque à la limite de ce qui est observable directement: un chat de Schrödinger mésoscopique, cela ne passe pas, malgré tout, inaperçu!.

\section{REFERENCES BIBLIOGRAPHIQUES}

ASPECT, Alain [1983]. Trois tests expérimentaux des inégalités de Bell par mesure de polarisation de photons, Thèse de doctorat ès-sciences physiques, Université Paris-Sud, Orsay, 1983.

BALIBAR, Françoise et LEVY-LeBLOND, Jean-Marc [1998]. Quantique. Tome 1 : Rudiments, Paris, 1998.

\footnotetext{
${ }^{56}$ Haroche, Brune, \& Raimond [1995].

57 Voir Paty [1999a, 2000d].

58 Pour une discussion, voir Paty [à paraître, b].
} 
BELL, John S. [1987]. Speakable and unspeakable in quantum mechanics, Cambridge University Press, Cambridge, 1987.

Ben Dov, Yoav [1988]. Problèmes de non-localité et de mesure en mécanique quantique, Thèse de doctorat en épistémologie et histoire des sciences, Université Paris 7-Denis Diderot/Paris 13, 1988.

BIMBOT, René et PATY, Michel [1996]. Vingt cinq années d'évolution de la physique nucléaire et des particules, in Yoccoz, Jean (éd.), Physique subatomique: 25 ans de recherche à l'IN2P3, la science, les structures, les hommes, Editions Frontières, Gif-sur-Yvette, 1996, p. 12-99.

BoHM, David [1980].Wholeness and the implicate order, Routledge and Kegan Paul, London, 1980.

BOHM, David \& HILEY, Basil J. [1993]. The Undivided Universe: An Ontological Interpretation Of Quantum Theory, London, Routledge, 1993.

BOHR, Niels [1935]. Can quantum-mechanical description of physical reality be considered complete ?, Physical Review 48, 1935, 696-702.

BOHR, Niels [1958]. Atomic physics and human knowledge, New York, Wiley, 1958 ; trad. fr. par Edmond Bauer et Roland Omnès, Physique atomique et connaissance humaine, Paris, Gauthier-Villars, nlle éd. établie par Catherine Chevalley, Paris, Gallimard, 1991.

CASSIRER, Ernst [1910]. Substanzbegriff und Funktionsbegriff, Bruno Cassirer, Berlin, 1910. Trad. angl., Substance and function, in Cassirer 1923 (éd.1953), p.1-346.

CASSIRER, Ernst [1921]. Zur Einstein'schen Relativitätstheory, Bruno Cassirer, Berlin, 1921. Trad. angl., Einstein's theory of relativity considered from the epistemological standpoint, in Cassirer 1923 (ed. 1953), p. 347-460.

CASSIRER, Ernst [1923]. Substance and function and Einstein's theory of relativity. Trad. angl. par William Curtis Swabey and Mary Collins Swabey, Open Court, Chicago, 1923; Dover, New York, 1953 [édition utilisée].

COMTE, Claude [1998]. Les probabilités quantiques sont-elles géométriques ?, in Flament, Dominique (éd.), Histoire de géométries, Textes du Séminaire de 1997, Document de travail, Maison des Sciences de l'Homme, Paris, 1998, p. 1-38.

CORNELl, Eric \& WIEMANN, Carl [1998]. The Bose-Einstein condensate, Scientific American, $\mathrm{n}^{\circ} 3$ (march), 1998, 26-31. Trad. fr., La condensation de Bose-Einstein, Pour la Science, n²47, mai 1998, 92-97.

DARRIGOL, Olivier [1992]. From c-Numbers to q-Numbers. The classical Analogy in the History of Quantum Theory, University of California Press, Berkeley, 1992.

DESCARTES, René [vers 1628]. Regulae ad directionem ingenii, in Descartes, Opuscula Posthuma, Amsterdam, 1701; in Oeuvres de Descartes, publiées par Charles Adam et Paul Tannery, 11 volumes (1ère éd., 1896-1913); nouvelle 
édition révisée, 1964-1974 ; ré-éd., 1996.: vol. 10, p. 349-486 ; trad. en français par J. Sirven, Règles pour la direction de l'esprit, Vrin, Paris, 1970.

DINER, Simon \& GUNZIG, Edgar [1998]. Univers du tout et du rien, Editions de l'Université de Bruxelles, Bruxelles, 1998.

DIRAC, Paul A. M. [1930]. The Principles of Quantum Mechanics, Clarendon Press, Oxford, 1930. 4th ed., 1958. Trad. fr. par Alexandre Proca et Jean Ullmo, Les principes de la mécanique quantique, Presses Universitaires de France, Paris, 1931.

EINSTEIN, Albert [1933]. On the method of theoretical physics, The Herbert Spencer lecture, Oxford, june 10, 1933 ; repris in Einstein [1954], p. 263-270. Original en allemand : Zur Methodik der theorischen Physik, in Einstein [1934a], ed. 1960, p. 113-119. Trad. fr., Au sujet de la méthode de la physique théorique, in Einstein [1934b], p. 163-173; autre trad. fr., Sur la méthodologie de la physique théorique, in Oeuvres, vol. 5 : Science, Ethique, Philosophie, Seuil, Paris, 1991, p. 102-107.

EINSTEIN, Albert [1934a]. Mein Weltbild, Querido, Amsterdam, 1934. Ré-édition (modif. et augm. de textes plus récents), Herausgeben von Carl Seelig, EuropaVerlages, Zurich, 1953 ; Ullstein Bücher, West-Berlin, 1960.

EINSTEIn, Albert [1934b]. Comment je vois le monde, Flammarion, Paris, 1934. (Trad. fr., par le Colonel Cros, de Einstein [1934a]). Nouvelle trad. par Maurice Solovine, Flammarion, Paris, 1958. Nouvelle trad. par Régis Henrion, Flammarion, Paris, 1969.

EINSTEIN, Albert [1936]. Physik und Realität, Franklin Institute Journal, CCXXI, 1936, 313-347. Trad. angl., Physics and reality, Franklin Institute Journal, CCXXI, 1936, 349-382 ; également in Einstein [1954], p. 283-315. Trad. fr., Physique et réalité, in Einstein [1952], p. 66-108. Autre tr. fr., Physique et réalité, in Einstein [1989-1993], vol. 5 : Science, Ethique, Philosophie, p. 125-151.

EINSTEIN, Albert [1940]. Considerations concerning the fundaments of theoretical physics, Science, n.s., XCI, 1940 (may 24), 487-492; repris sous le titre The fundaments of theoretical physics, in Einstein [1954], p. 315-326. Trad. fr., Les fondements de la physique théorique, in Einstein [1952], p. 109-123.

EINSTEIN, Albert [1948]. Quantenmechanik und Wirklichleit, Dialectica 2, 1948, 35-39. Trad. fr., Mécanique quantique et réalité, in Einstein [1989], p. 244-249.

EINSTEIN, Albert [1952]. Conceptions scientifiques, morales et sociales, trad. fr. par Maurice Solovine, Flammarion, Paris, 1952. Ré-éd. (abrégée), trad. fr. revue par Daniel Fargue, Flammarion, Paris, 1990.

EInstein, Albert [1954]. Ideas and Opinions, transl. by Sonja Bergmann, Crown, New-York, 1954. Ré-éd. Laurel, New-York, 1981.

EInstein, Albert [1987-]. The Collected Papers of Albert Einstein, ed. by John Stachel et al., puis par M. Klein et al., Princeton University Press, Princeton, New Jersey, 8 vols. parus de 1987 à 199 ? 
EINSTEIN, Albert [1989-1993]. Oeuvres choisies, trad. en fr. sus la dir. de Françoise Balibar, Seuil, Paris, 1989-1993, 6 vols.

EINSTEIN, Albert; PODOLSKY, Boris \& Rosen, Nathan [1935b]. Can quantummechanical description of physical reality be considered complete ?, Physical Review , ser. 2, XLVII, 1935, 777-780. Trad. fr., Peut-on considérer que la mécanique quantique donne de la réalité physique une description complète ?, in Einstein [1989-1993], vol. 1: Quanta : Mécanique statistique et physique quantique., p. 224-230.

EINSTEIN, Albert \& BORN, Max [1969]. Briefwechsel 1916-1955, Nymphenburger Verlagshandlung, München, 1969. Trad. fr. par Pierre Leccia, Correspondance 1916-1955, commentée par Max Born, Seuil, Paris, 1972.

FAYET, Pierre [1985]. La supersymétrie et l'unification des interactions fondamentales, La Recherche 19, ${ }^{\circ}$ 197, mars 1988, 334-345.

FrEIRE Jr., Olival [1995]. A emergencia da totalidade. David Bohm e a controversia dos quanta, Tese de doutorado, Dpto de História, Universidade de São Paulo, 1995.

GRANGER, Gilles-Gaston [1999]. La pensée de l'espace, Odile Jacob, Paris, 1999.

GRANGIER, Philippe [1986]. Etude expérimentale de propriétés non-classiques de la lumière; interférences à un seul photon, Thèse de doctorat ès-sciences physiques, Université Paris-Sud, Orsay, 1986.

Griffin, A, SNOKE, D.W. \& STRINGARI, S. (eds.) [1995]. Bose-Einstein condensation, Cambridge University Press, 1995.

Grunbaum, Adolf [1963]. Philosophical problems of space and time, Knopf, New York, 1963. Second, enlarged, ed., Reidel, Dordrecht, 1973.

Guenault, M. [1756]. Etendue, in d'Alembert, Jean \& Diderot, Denis (éds.), Encyclopédie ou Dictionnaire raisonné des sciences, des arts et des métiers, vol. 6, (1756), p. 171-174.

HAROCHE, Serge, BRUNE, M. \& RAIMOND, Jean-Michel [1997]. Experiments with single atoms in a cavity : entanglement, Schrödinger's cats and decoherence, Philosophical Transactions of the Royal Society of London A, 1997, 355, 23672380 .

HELMHOLTZ, Hermann von [1921]. Schriften zur Erkenntnistheorie, herausgegeben und erlaütert von Paul Hertz und Moritz Schlick, Springer, Berlin, 1921.1978. Trad. angl. : Epistemological writings. The Paul Hertz/Moritz Schlick centenary edition of 1921 with notes and commentary by the editors; newly transl. by Malcolm F. Lowe. Ed. with an Intoduction and bibliography by R.S. Cohen and Y. Elkana, Reidel, Dordrecht and Boston, 1978.

HILBERT, David [1899]. Grundlagen der Geometrie (1899); $7^{\text {è }}$ éd. avec appendices, Teubner, Berlin, 1930 ; trad. fr. (partielle) par P. Rossier, Les fondements de la géométrie, Dunod, Paris, 1971. 
ITZYKSON, Claude et ZUBER, J.B. (1980). Quantum field theory, Mc GrawHill, New York, 1980.

KANT, Immanuel [1781]. Critik der reinen Vernunft, J.F. Hartknoch, Riga, 1781 ; 2 è ed., 1787. Trad. fr. par Alexandre J.L. Delamarre et François Marty, Critique de la raison pure, in Kant, Emmanuel, Oeuvres philosophiques, vol. 1, Gallimard, Paris, 1980, p. 705-1470.

KOUNEIHER, Joseph [1998]. Epistémologie et histoire récente de la gravitation quantique, Thèse de doctorat en épistémologie et histoire des sciences, Université Paris 7-Denis Diderot, 1998.

LANGEVIN, Paul [1934]. La notion de corpuscules et d'atomes, Hermann, Paris, 1934.

LeITE LOPES, José \& EsCOUBES, Bruno [1995]. Sources et évolution de la physique quantique. Textes fondateurs, Masson, Paris, 1995.

LeITE LOPES, José \& PATY, Michel (eds.) [1977]. Quantum Mechanics Half a Century Later, Reidel, Dordrecht, 1977

LEVY-LEBLOND, Jean-Marc [1977a]. Towards a Proper Quantum Theory, in Leite Lopes, José \& Paty, Michel (eds.), Quantum Mechanics Half a Century Later, Reidel, Dordrecht, 1977, -.

LEVY-Leblond, Jean-Marc [1977b]. The Picture of the Quantum World : from Duality to Unity, International Journal of Quantum Chemistry 12, supp. 1, 1977, 415-.

LEVY-LeBlond, Jean-Marc [1988]. Neither Waves, nor Particles, but Quantons, Nature 334, 1988, 6177.

LEVY-LEBLOND, Jean-Marc [1996]. Quantum Theory at Large, in Beltrametti, E. \& Lévy-Leblond, Jean-Marc (eds), Advances in Quantum Phenomena, Plenum, New York 1996.

MACH, Ernst [1883]. Die Mechanik in ihrer Entwicklung historisch-kritisch Dargellstellt, Leipzig, 1883; autres éditions augm. de 1888 à 1933. Trad. angl. (sur la 2 ème éd. allemande), par Thomas J. McCormack, The science of mechanics. A critical and historical exposition of its principles, Open Court, Chicago, 1893 ; autres éd. augm., de 1902 à 1960. Trad. fr. (sur la 4 ème éd. allemande) par E. Bertrand, La mécanique. Exposé historique et critique de son développement, Hermann, Paris, 1904; ré-éd., 1923.

MACH, Ernst [1905]. Erkenntnis und Irrtum. Skizzen zur Psychologie der Forschung, J. A. Barth, Leipzig, 1905; 2 ème éd. augm., 1906 ; autres éd.: 1917, 1920, 1926. Trad. fr. (abrégée, sur la 2 ème éd. allemande) par Marcel Dufour, La connaissance et l'erreur, Flammarion, Paris, 1908 ; ré-éd., 1922. Trad. angl. (sur la 5 ème éd. allemande) par Paul Foulkes et Thomas J. McCormack, Knowledge and error, Reidel, Dordrecht, 1976.

MACH, Ernst [1906]. Space and geometry, in the light of physiological, psychological and physical inquiry, transl. from the German by Thomas J. McCormack, Open Court, La Salle (Ill.), 1906. 
MeHrA, Jagdish \& RECHENBERG, Helmut [1982]. The Historical Development of Quantum Theory, vol. 4, Parts 1 and 2, Springer-Verlag, New-York, Berlin, 1992.

NeumanN, John von [1932]. Mathematische Grundlagen der Quantenmechanik, Springer, Berlin, 1932. Trad. fr. par Alexandre Proca, Les fondements mathématiques de la mécanique quantique, Librairie Alcan et Presses Universitaires de France, Paris, 1947.

PATY, Michel [1985d]. Symétrie et groupes de transformation dans les théories contemporaines de la matière : jalons épistémologiques, Colloque Abel-Galois, Lille, 21-25 février 1983, Première partie, Institut de Recherches de Mathématiques Avancées (IRMA), Lille, 1985, fasc 5.

PATY, Michel [1986b]. La non-séparabilité locale et l'objet de la théorie physique, Fundamenta Scientiae 7, 1986, 47-87.

PATY, Michel [1988a]. La matière dérobée. L'appropriation critique de l'objet de la physique contemporaine, Archives contemporaines, Paris, 1988, xx $+442 \mathrm{p}$.

PATY, Michel [1989a]. L'inséparabilité et la mesure des systèmes quantiques, in Jacob, André (dir.) Encyclopédie philosophique universelle, volume 1: André Jacob (dir.), L'Univers philosophique, Presses Universitaires de France, Paris, 1989, p. 1172-1177. Repris dans [1990a], chap. 12, p. 164-175.

PATY, Michel [1990]. L'analyse critique des sciences, ou le tétraèdre épistémologique (sciences, philosophie, épistémologie, histoire des sciences), L'Harmattan, Paris, 1990.

PATY, Michel [1993]. Einstein philosophe. La physique comme pratique philosophique, Presses Universitaires de France, Paris, 1993.

PATY, Michel [1994a]. Le caractère historique de l'adéquation des mathématiques à la physique, in Garma, Santiago; Flament, Dominique; Navarro, Victor (eds.), Contra los titanes de la rutina.- Contre les titans de la routine, Comunidad de Madrid/C.S.I.C., Madrid, 1994, p. 401-428.

PATY, Michel [1994b]. Sur l'histoire du problème du temps : le temps physique et les phénomènes, in Klein, Etienne et Spiro, Michel (éds.), Le temps et sa flèche, Editions Frontières, Gif-sur-Yvette, 1994, p. 21-58; 2è éd., 1995 ; Collection Champs, Flammarion, Paris, 1996, p. 21-58.

PATY, Michel [1995]. The nature of Einstein's objections to the Copenhagen interpretation of quantum mechanics, Foundations of physics 25, 1995, $\mathrm{n}^{\circ} 1$ (january), 183-204.

PATY, Michel [1997e]. «Mathesis universalis» e inteligibilidad en Descartes, Trad. en español por Martha Cecilia Bustamente, in Albis, Victor R. ; Charum, Jorge ; Sanchez, Clara Helena ; Serrano, Gonzalo (eds.), Memorias del Seminario en conmemoración de los 400 años del nacimiento de René Descartes, Academia Colombiana de Ciencias Exactas, Físicas y Naturales, Coleccion Memorias, ${ }^{\circ} 9$, Bogotá, 1997, p. 135-170. Version en portugais: «Mathesis universalis» e inteligibilidade em Descartes, Trad. em português por Maria Aparecida CorrêaPaty, Cadernos de História e Filosofia da Ciência (Campinas), Série 3, vol. 8, 
1998 (n¹, jan.-jun.), 9-57. Egalement, in Seminário sobre O Cartesianismo, Centro de Estudos de História e Filosofia da Ciência, Centros de Investigação da Universidade de Évora, Evora, Portugal, 2000, p. 145-200. Original en français : «Mathesis universalis» et intelligibilité chez Descartes, in Karine Chemla, Siegmund Probst, Agnès Erdély et Antonio Moretto (eds.), Ceci n'est pas un festschrift pour Imre Toth (29.12.1996), à paraître.

PATY, Michel [1998a]. Le vide matériel, ou : La matière crée l'espace, in Diner, Simon \& Gunzig, Edgard (éds.), Univers $d u$ tout et $d u$ rien, Editions de l'Université de Bruxelles, Bruxelles, 1998, p. 22-44.

PATY, Michel [1998b]. Les trois dimensions de l'espace et les quatre dimensions de l'espace-temps in Flament, Dominique (éd.), Dimension, dimensions I, Série Documents de travail, Fondation Maison des Sciences de l'Homme, Paris, 1998, p. 87-112.

PATY, Michel [1999a]. Are quantum systems physical objects with physical properties?, European Journal of Physics 20, 1999 (november), 373-388. (Special issue on « Unsolved problems of physics ».)

PATY, Michel [1999b]. La création scientifique selon Poincaré et Einstein, in Serfati, Michel (éd.), La recherche de la vérité, Coll. L'Ecriture des Mathématiques, ACL-Editions du Kangourou, Paris, 1999, p. 241-280.

PATY, Michel [2000a]. Einstein et la pensée de la matière, in Monnoyeur, Françoise (éd.), La matière des physiciens et des chimistes, Le Livre de poche, Hachette, Paris, 2000, p. 213-252.

PATY, Michel [2000b]. Cosmologie et matière quantique, in Seidengart, Jean et Sczeciniarz, Jean-Jacques (éds.), Cosmologie et philosophie. Hommage à Jacques Merleau-Ponty, numéro spécial de Epistémologiques, philosophie, sciences, histoire. Philosophy, science, history (Paris, São Paulo) 1, $\mathrm{n}^{\circ} 1-2$, janvier-juin 2000, 219-249.

PATY, Michel [2000c]. Interprétations et significations en physique quantique, Revue Internationale de Philosophie (Bruxelles), n²12, 2 (juin) 2000, 199-242.

PATY, Michel [2000d]. The quantum and the classical domains as provisional parallel coexistents, Synthese (Kluwer, Dordrecht/Boston), 125, $\mathrm{n}^{\circ} 1-2$ (Octobernovember), 2000, 179-200 (in French, Steven; Krause, Décio ; Doria, Francisco Antonio (eds.), Festschrift in honor of Newton da Costa on the occasion of his seventieth birthday).

PATY, Michel [2001a]. Physical quantum states and the meaning of probability, as Chapter 14, in Galavotti, Maria Carla, Suppes, Patrick \& Costantini, Domenico, (eds.), Stochastic Causality, CSLI Publications (Center for Studies on Language and Information), Stanford (Ca, USA), 2001, p. 235-255.

PATY, Michel [2001b]. La notion de grandeur et la légitimité de la mathématisation en physique, in Espinoza, Miguel (éd.), De la science à la philosophie : Hommage à Jean Largeault, L'Harmattan, Paris, 2001, p. 247-286.

PATY, Michel [2002]. La physique quantique ou l'entraînement de la forme 
mathématique sur la pensée physique, in Mataix, Carmen y Rivadulla, Andrés (eds.), Física cuantica y realidad. Quantum physics and reality, Editorial Complutense, Madrid, 2002, p. 97-134.

PATY, Michel [à paraître, a] Einstein, les quanta et le réel. Critique et construction théorique.

PATY, Michel [à paraitre, b]. The concept of quantum state : new views on old phenomena, in Cohen, Robert S.; Howard, Don ; Renn, Jürgen ; Sarkar, Sahotra \& Shimony, Abner (eds.), John Stachel Festschrift, Boston Studies in the Philosophy and History of science, Kluwer, Dordrecht, à paraître, in press. (Vers. fr. non publiée : Le concept d'état quantique : un nouveau regard sur d'anciens phénomènes.)

PATY, Michel [à paraître, c]. Construction d'objet et objectivité en physique quantique, Intervention à la Journée sur la question des "conditions d'application" de l'objectivisme et du constructivisme à la description de certains phénomènes physiques ou sociaux, Université Paris 7-Denis Diderot (Centre Javelot), Paris, mardi 22 mai 2001.

POINCARE, Henri [1902]. La science et l'hypothèse, Flammarion, Paris, 1902 ; 1968.

POINCARE, Henri [1905]. La valeur de la science, Flammarion, Paris, 1905 ; 1970.

POINCARE, Henri [1908]. Science et méthode, Flammarion, Paris, 1908.

PoInCARE, Henri [1913]. Dernières pensées, Flammarion, Paris, 1913 ; réed. 1963.

Reichenbach, Hans [1928]. Philosophie der Raum Zeit Lehre, de Gruyter, Berlin, 1928. Trad. angl. par Maria Reichenbach et John Freund, The philosophy of space and time, Dover, New York, 1957.

REICHENBACH, Hans [1978]. Selected writings, edited by Robert S. Cohen and Maria Reichenbach, 2 vols, Reidel, Dordrecht, 1978.

ROSENFELD, Léon [1979]. Selected papers, edited by Robert S. Cohen and John Stachel, Reidel, Dordrecht, 1979.

RIEMANN, Bernhard [1854]. Ueber die Hypothesen, welche der Geometrie zugrunde liegen [Mémoire présenté le 10 juin 1854 à la Faculté philosophique de Göttingen], Abhandlungender königlischen Gessellschaft der Wissenschaften zu Göttingen, vol. 13, 1867 ; Trad. fr. par J. Houel, "Sur les hypothèses qui servent de fondement à la géométrie", in B.R., Oeuvres mathématiques, trad. fr. par L. Laugel, avec une préface de M. Hermitte et un essai de M. Félix Klein, Paris, 1898 ; nouveau tirage, Paris, 1968., p. 280-299. Trad. angl. in B. R., Collected works, ed. par H. Weber et R. Dedekind, with supplement by M. Noether and W. Wirtinger, New York, 1953.

SCHILPP, Paul-Arthur [1949]. Albert Einstein : philosopher-scientist, The library of living philosophers, Open Court, Lassalle (Ill.), 1949. Ré-ed. 1970. Trad. en allemand, Albert Einstein als Philosoph und Naturforscher, Kohlhammer Verlag, 
Stuttgart, 1955 (textes originaux d'Einstein et de Pauli).

SCHRÖDINGER, Erwin 1950. Space-time structure, Cambridge University Press, Cambridge, 1950 ; repr. with corrections, $1960 ; 1988$.

SEIDENGART, Jean et ScZECINIARZ, Jean-Jacques (éds.) [2000]. Cosmologie. En hommage à Jacques Merleau-Ponty, numéro spécial de Epistémologiques. Philosophie, sciences, histoire. Philosophy, Science, History (Paris, São Paulo) 1, (n¹-2), 2000.

WEYL, Hermann [1918]. Raum, Zeit, Materie. [1ère édition, cours professé à l'Ecole Polytechnique fédérale de Zurich en 1917.] 3ème édition remaniée, 1919. 4ème édition, augmentée, 1921. Trad. fr. sur la quatrième édition allemande, par Gustave Juvet et Robert Leroy, Temps, espace, matière. Leçons sur la théorie de la relativité générale, Blanchard, Paris, 1922. Nouveau tirage augmenté de commentaires par Georges Bouligand, Blanchard, Paris, 1958 ; 1979.

WEYL, Hermann [1928]. Gruppentheorie und Quantenmechanik, Hirzel, Leipzig, 1928 (2ème éd. 1931). Trad. angl. par H. P. Robertson, The theory of groups and quantum mechanics, Methuen, London, 1931 ; Dover, New York, 1950.

WhEELER, John A. \& ZUREK, Wojcieh H. (eds.) [1983]. Quantum theory of measurement, Princeton University Press, Princeton, 1983.

ZAHAR, Elie [1994]. Against the (alleged) divorce between science and philosophy. Comments on M. Paty's La matière dérobée, Rivista di Storia della Scienza (Roma), serie II, volume 2, n²1, giugno 1994, 191-208. 